\title{
Particle Habit Imaging Using Incoherent Light: A First Step toward a Novel Instrument for Cloud Microphysics
}

\author{
ROLAND SCHÖN AND MARTIN SCHNAITER \\ Institute for Meteorology and Climate Research, Karlsruhe Institute of Technology, Karlsruhe, Germany \\ ZBIGNIEW ULANOWSKI \\ Science and Technology Research Institute, University of Hertfordshire, Hatfield, United Kingdom \\ CARL SCHMITT \\ National Center for Atmospheric Research, Boulder, Colorado
}

\begin{abstract}
Stefan Benz, Ottmar Möhler, Steffen Vogt, Robert Wagner, And Ulrich Schurath
Institute for Meteorology and Climate Research, Karlsruhe Institute of Technology, Karlsruhe, Germany
\end{abstract}

(Manuscript received 20 January 2010, in final form 31 August 2010)

\begin{abstract}
The imaging unit of the novel cloud particle instrument Particle Habit Imaging and Polar Scattering (PHIPS) probe has been developed to image individual ice particles produced inside a large cloud chamber. The PHIPS produces images of single airborne ice crystals, illuminated with white light of an ultrafast flashlamp, which are captured at a maximum frequency of $\sim 5 \mathrm{~Hz}$ by a charge-coupled device (CCD) camera with microscope optics. The imaging properties of the instrument were characterized by means of crystalline sodium hexafluorosilicate ice analogs, which are stable at room temperature. The optical resolving power of the system is $\sim 2 \mu \mathrm{m}$. By using dedicated algorithms for image processing and analysis, the ice crystal images can be analyzed automatically in terms of size and selected shape parameters. PHIPS has been operated at the cloud simulation chamber facility Aerosol Interaction and Dynamics in the Atmosphere (AIDA) of the Karlsruhe Institute of Technology at different temperatures between $-17^{\circ}$ and $-4^{\circ} \mathrm{C}$ in order to study the influence of the ambient conditions, that is, temperature and ice saturation ratio, on ice crystal habits. The area-equivalent size distributions deduced from the PHIPS images are compared with the retrieval results from Fourier transform infrared (FTIR) extinction spectroscopy in case of small $(<20 \mu \mathrm{m})$ and with single particle data from the cloud particle imager in case of larger $(>20 \mu \mathrm{m})$ ice particles. Good agreement is found for both particle size regimes.
\end{abstract}

\section{Introduction}

Ice crystals in cirrus have been shown to take numerous different shapes. In addition to simple columns and plates, which mainly represent the hexagonal lattice of ice, hollow columns, rosettes, and aggregates are found (Heymsfield and Miloshevich 2003). The morphology of ice crystals in cirrus clouds has a direct impact on the

Corresponding author address: Martin Schnaiter, Hermann-vonHelmholtz-Platz 1, Institute for Meteorology und Climate Research, Karlsruhe Institute of Technology, 76344 Eggenstein-Leopoldshafen, Germany.

E-mail: martin.schnaiter@kit.edu radiative forcing. For different ice crystal habits, the radiative forcing of cirrus with moderate optical thickness may vary over $100 \mathrm{~W} \mathrm{~m}^{-2}$ (Wendisch et al. 2005). The radiative properties of a single ice crystal are dependent on the particle's size, shape, and orientation. Cirrus clouds reflect incoming shortwave solar radiation and absorb outgoing longwave terrestrial radiation. Depending on the ratio of these two effects, a positive or negative radiative forcing on the atmosphere may result. Ice clouds consisting of smaller crystals $(<50 \mu \mathrm{m})$ have a cooling effect, whereas for bigger crystals the warming effect dominates (Zhang et al. 1999).

Imaging of atmospheric ice crystals and the study of their morphology has been the subject of ongoing 
research for more than $50 \mathrm{yr}$ (Cannon 1976; Bailey and Hallet 2004). The first experiments were carried out by holding Plexiglas plates, freshly coated with Zapon lacquer, out of an aircraft (Weickmann 1945, 1949). Ice crystals impacting the plate leave impressions that can be examined by means of a microscope after the flight. This replica technique is still used by the video ice particle sampler (VIPS; McFarquhar and Heymsfield 1996). In this instrument the lacquer-coated plate is replaced by a moving oil-coated film. Ice crystals stick to the oil on the film, which then moves through the field of view of a video camera. After the crystals are imaged the particles are scraped off and the oil film is renewed. A similar technique is used by the cloudscope (Arnott 1998) where the crystals impact a sapphire window that is imaged continuously. The VIPS instrument has been used in ice cloud characterization studies at the Aerosol Interaction and Dynamics in the Atmosphere (AIDA) cloud simulation chamber of the Karlsruhe Institute of Technology (KIT). Although the impaction technique ensures that the particles are captured in the depth of field of the imaging system, a major disadvantage is the fact that the random orientation of the crystals is lost. This is because the crystals tend to settle with their largest facet on the impaction surface. This leads to oversizing when the crystals are compared to measurements of randomly oriented particles that are present in the atmosphere as well as in the turbulently mixed AIDA volume. The oversizing problem can be avoided by using nonintrusive imaging methods for airborne cloud particles. Such a concept has been implemented in the cloud particle imager [CPI; Stratton Park Engineering Company (SPEC), Inc.] research aircraft instrument, which has been deployed in AIDA experiments as well (Field et al. 2006). The CPI captures magnified bright field images of airborne cloud particles on an 8-bit chargecoupled device (CCD) camera (Lawson et al. 2001). Because a pulsed laser diode that emits coherent light is used for illuminating individual particles, diffraction patterns are often seen in CPI data, which complicates the determination of the particle size, especially for out-offocus and small particles.

Here, we present another nonintrusive imaging setup that uses the white light emission of an ultrafast flashlamp for illumination. The imaging system described in the following sections is part of the novel dual-imaging and laser-scattering instrument, the particle habit imaging and polar scattering (PHIPS), which is currently under development at KIT. PHIPS is the first instrument that combines a particle imager with a polar nephelometer for the simultaneous measurement of the angular scattering function of the imaged particle (early versions of the CPI tried to do this as well). The main differences between the imager setup of PHIPS and the CPI are (i) the illumination by incoherent light that generates diffraction pattern-free images with smooth-edged gradients, and (ii) the use of a trigger detector system that effectively confines the depth from which particles are imaged to the depth of field of the imaging system. Section 2 gives a detailed description of the imaging system that is implemented in PHIPS to image individual cloud particles from one direction. A brief outline of the instrumentation used at the cloud chamber AIDA and the experimental procedures are also given in section 2 . The image processing and analyzing procedures are presented in section 3 , and the results are discussed in section 4. A summary and an outlook on the final PHIPS instrument are given in section 5 .

\section{Experimental}

\section{a. Setup of the imaging part of PHIPS}

Figure 1 shows a schematic drawing of the complete setup, including the sample volume. The microscope unit comprises a black-and-white CCD camera (a PCO Pixelfly QE: $1392 \times 1024$ pixels, 12 bit, and $6.45 \mu \mathrm{m} \times$ $6.45 \mu \mathrm{m}$ pixel size), a macrozoom lens (an Intravision LVM 1200: 2 times magnification tubus), and a flashlamp (a HSPS Nanolite KL-K). The CCD camera is connected to a computer that is used for controlling the data acquisition and for storing the recorded images. At 8 times magnification, the field of view of the microscope has a size of $1.1 \mathrm{~mm} \times 0.8 \mathrm{~mm}$. One pixel in the recorded image thus corresponds to a square with $0.8-\mu \mathrm{m}$-long edges in the object plane. This is smaller than the optical resolving power of the microscope, which is $\sim 2 \mu \mathrm{m}$. The resolving power has been determined by imaging thin tungsten wires of known thickness. The implemented flashlamp emits short pulses of broadband white light in the 200-800-nm range with a duration of 9 ns. A short pulse duration is required to avoid the blurring of the images resulting from the movement of the particles with a velocity of $\sim 2 \mathrm{~m} \mathrm{~s}^{-1}$ through the object plane of the microscope unit (see the description of the instrument chamber below). Recording an image is triggered by means of an optical particle detector. This detector is sensitive to scattered light that is emitted by particles moving through the object plane of the microscope unit. A photoreceiver (a Femto OE-200-Si-SMA) converts the optical signal to a transistor-transistor logic (TTL) pulse. The rising edge of this pulse is then used for triggering the camera shutter and the flashlamp. Because the camera and the flashlamp have different latencies $(25 \mu \mathrm{s}$ of the CCD versus $5 \mu$ s of the flashlamp), the flash trigger is delayed by at least $25 \mu \mathrm{s}$ with respect to the CCD exposure trigger. 


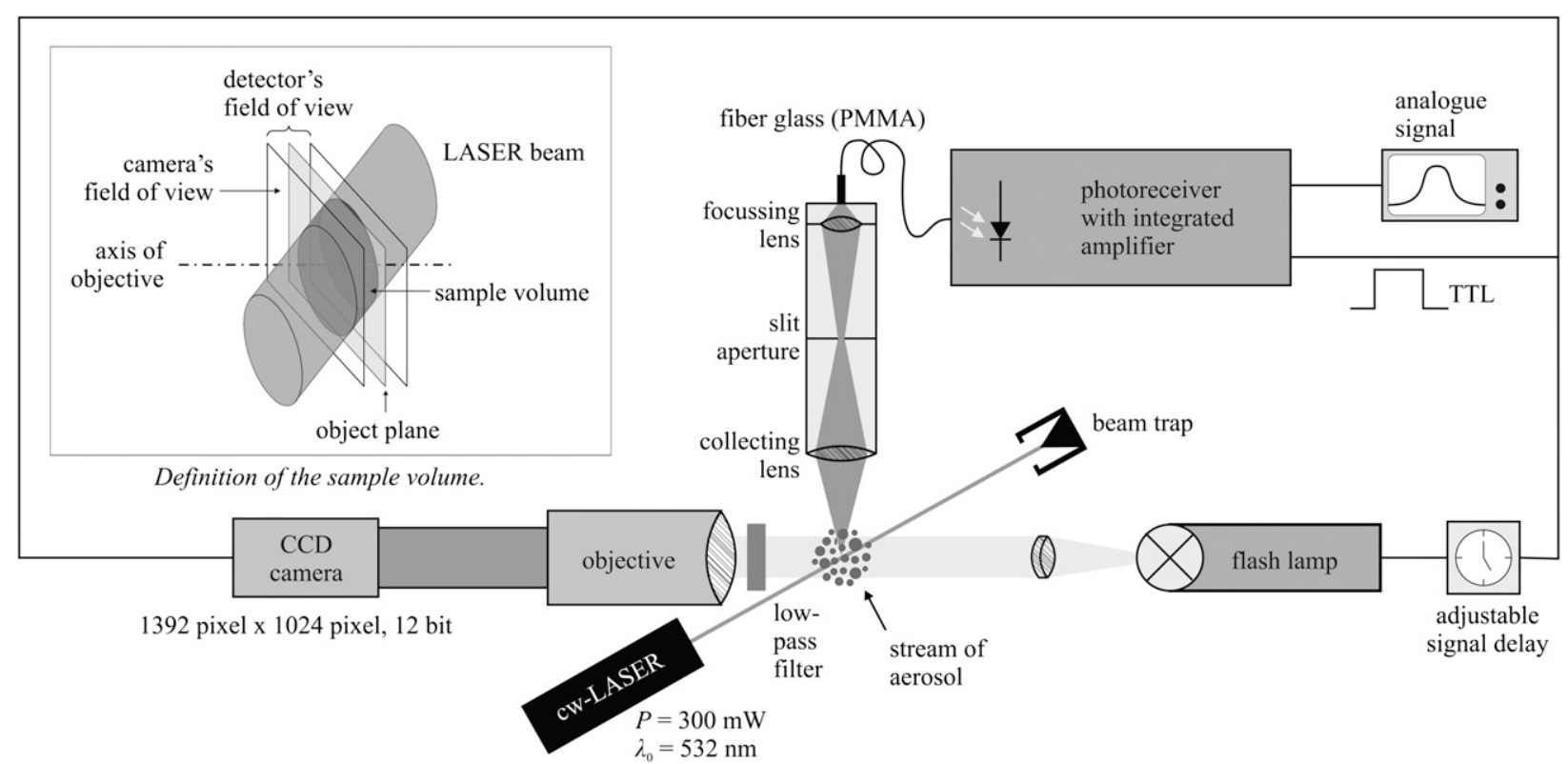

FIG. 1. Experimental setup and definition of the sample volume. The hydrometeors move perpendicular to the plane of projection.

To detect a particle, the sample volume is illuminated by a green continuous wave $(\mathrm{CW})$ laser beam (a Coherent Compass 415M-300: wavelength in vacuum, $532 \mathrm{~nm}$; emitted power, $300 \mathrm{~mW}$ ) under an angle of $29^{\circ}$ with respect to the optical axis of the microscope unit. The diameter of the beam is $\sim 0.5 \mathrm{~mm}$, which is less than the length of the shorter side of the field of view of the microscope. In this way it is ensured that sufficiently small particles that enter the beam and scatter light reside completely in the field of view of the microscope. The optics of the particle detector are oriented perpendicular to the optical axis of the microscope and the laser beam. The intersection point of the optical axis of the microscope with the axis of the detector optics matches the focus of the microscope. By using a $200-\mu \mathrm{m}$-wide slit aperture in combination with a 2 times objective lens in the particle detector optics, the particle detector probes scattered light from a $100-\mu \mathrm{m}$-wide area within the object depth of the microscope. Because the slit aperture is oriented perpendicular to the optical axis of the microscope, the particle detector is effectively sensitive to particles that pass the object plane within the depth of field $( \pm 50 \mu \mathrm{m})$ of the microscope. Thereby, the instrument only selects those particles that will likely results in a sharp image. The laser beam and slit aperture define a sample volume that has the shape of a tilted cylinder as shown in the inset in Fig. 1. An eyepiece lens projects the slit aperture onto the end of an optical fiber that is connected to the photoreceiver. To prevent the scattered laser light from disturbing the captured image, a low-pass filter is mounted in front of the objective, which only transmits light with a wavelength shorter than $500 \mathrm{~nm}$. The objective itself is transparent to light, with a wavelength longer than $400 \mathrm{~nm}$.

The sample volume resides in the center of a vacuumsealed chamber made of stainless steel. Hydrometeors are pumped vertically through the sample volume via an entrance and an outlet tube with an inner diameter of $10 \mathrm{~mm}$. The outlet tube is connected to a vacuum system providing a constant mass flow of 10 standard liters per minute. This results in a laminar flow with an average airspeed of $\sim 2 \mathrm{~m} \mathrm{~s}^{-1}$ inside the sampling tube. The particles move perpendicular to the plane spanned by the laser beam and the optical axis of the particle detector and parallel to the plane spanned by the laser beam and the optical axis of the microscope. The laser beam and the particle flow are separated by an angle of $90^{\circ}$. Note that particles move horizontally through all of the PHIPS images presented in section 4.

\section{b. Experiments at the AIDA cloud chamber}

The stainless steel housing of PHIPS containing the sample volume is connected to the bottom of the AIDA chamber that is depicted in Fig. 2. Here, the AIDA instrumentation and the experimental procedure will only be described briefly; a more detailed description can be found in Möhler et al. (2003). AIDA can be operated as an expansion cloud chamber with thermodynamic conditions that closely mimic the conditions in rising air parcels in the atmosphere. In this way the chamber is used for investigation of the aerosol-driven ice nucleation and growth processes in atmospheric mixed-phase 


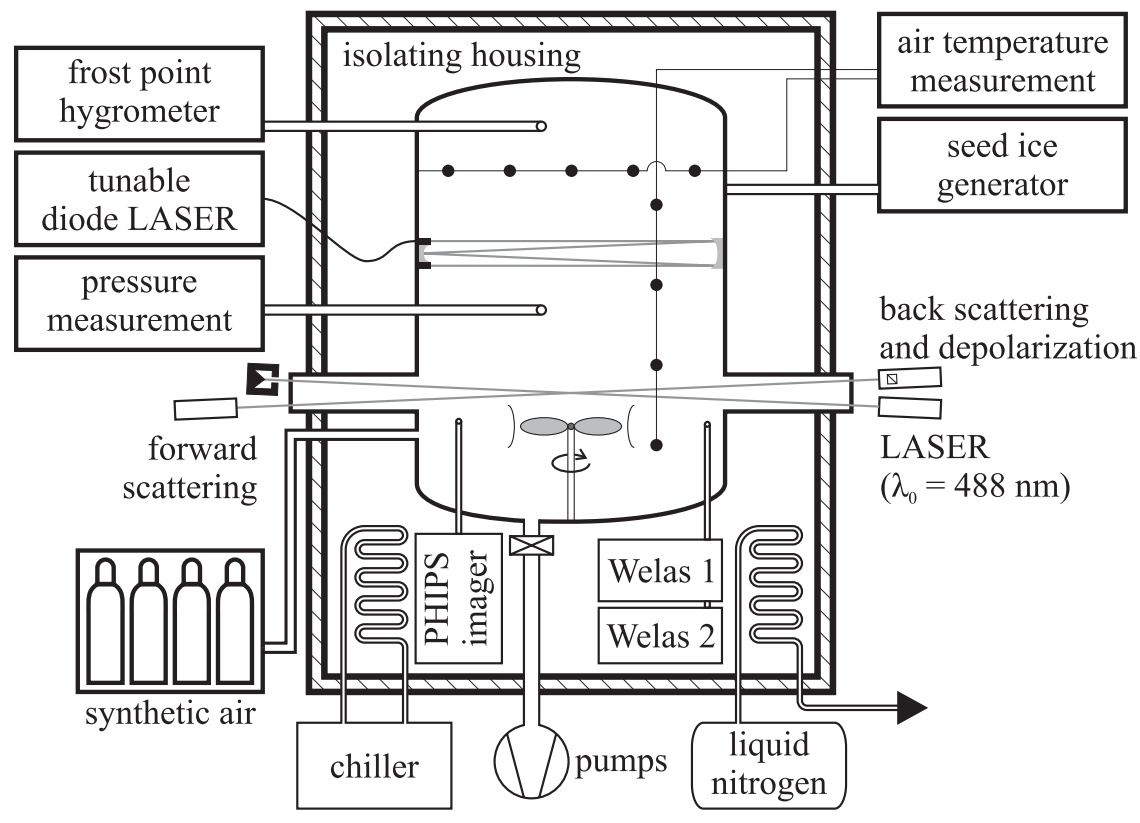

FIG. 2. Schematic of the experimental facility AIDA. The complete instrumentation is much more complex. For the sake of clarity, only the instruments that are relevant for this work are depicted, except for the CPI, which was operated similarly to the PHIPS in IN08_42.

and ice clouds. In addition to the ice nucleation studies, ice particle characterization experiments are performed in the chamber, where small, externally generated seed ice crystals are grown in the chamber at controlled temperature and saturation conditions [details can be found in Amsler et al. (2009)]. This type of experiment was applied in the present study to characterize the imaging properties of the PHIPS instrument.

The total water concentration in the chamber, that is, interstitial plus cloud water, is measured ex situ with a high precision frost-point hygrometer (a MBW Calibration 373 option LX). The hygrometer is connected to the chamber via a heated sampling tube to evaporate droplets and small ice crystals. Interstitial water vapor is measured in situ by means of a tunable diode laser (TDL) absorption spectrometer (Ebert et al. 2005). In the present study, the data obtained from the TDL measurements are calibrated by means of the frost-point hygrometer: the partial pressure deduced from the TDL absorption data is adjusted to the hygrometer data in a time period prior to the experiment when the chamber is free of cloud particles and humidified to near-ice-saturated conditions (Möhler et al. 2006).

Particle number concentration and size distribution of the hydrometeors inside the AIDA chamber can be measured by means of two optical particle counters (the Palas GmbH Welas), which are described in detail in Benz et al. (2005). The principle of operation is based on measuring a pulse of scattered white light that is emitted by a high pressure Xenon arc lamp. Two versions of the instrument are available. One is capable of discriminating particles with a diameter between 0.7 and $40 \mu \mathrm{m}$, the other one has a measuring range between 2.3 and $104 \mu \mathrm{m}$.

The self-built laser light-scattering and depolarization instrument SIMONE probes ensembles of aerosol and hydrometeor particles that reside in the center of the chamber. Detailed descriptions of the instrument including recent applications and results can be found in Wagner et al. (2009), Amsler et al. (2009), and M. Schnaiter et al. (2011, unpublished manuscript). The instrument analyzes polarized laser light that is scattered by the aerosol and cloud particles in near-forward direction at an angle of $1.8^{\circ}$ and in near-backward direction at an angle of $178.2^{\circ}$. In the latter case the light detection is polarization resolved. Therefore, it is possible to distinguish between spherical (droplets) and aspherical (ice) particles by analyzing the depolarization ratio, which is defined as the ratio of the perpendicular $I_{\perp}$ and parallel $I_{\|}$components (with respect to the incident polarization) of the backscattered intensity.

Figure 3 shows the setup that was used to generate micrometer-sized ice crystals in the ice particle characterization experiments described in section 4 . The basic principle of this seed ice generator is that a cold and dry ammonium sulfate aerosol flow is turbulently mixed with warm, humid, and particle-free air in a small chamber that is maintained at about $-63^{\circ} \mathrm{C}$. A number concentration 


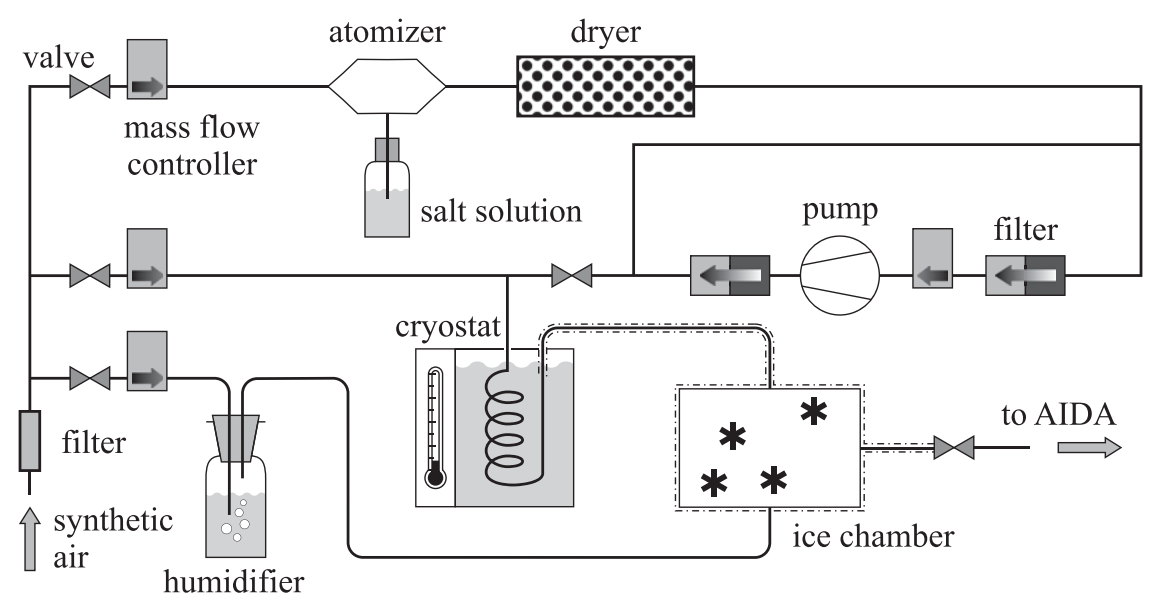

FIG. 3. Schematic of the setup that is used for seed ice generation.

of about $10^{4} \mathrm{~cm}^{-3}$ micrometer-sized ice crystals was obtained downstream of the mixing chamber by properly adjusting the flow rates and composition of the dry ammonium sulfate aerosol and the humid particle-free air. This was sufficient enough to enrich the AIDA volume to an ice particle number concentration of about $10 \mathrm{~cm}^{-3}$ within several minutes. Close to the injection position on the inner wall of the AIDA chamber, a nozzle is installed that can be used to spray ultrapure water droplets into the chamber volume. In this way, supercooled water droplets can be supplied at temperatures above $-35^{\circ} \mathrm{C}$, which serve as a reservoir of water vapor for the growth of the ice crystals via the Bergeron-Findeisen mechanism.

\section{PHIPS particle image analysis}

The analysis of the PHIPS images delivers geometric quantities and dimensionless shape parameters of the particles. The sphere-equivalent diameter

$$
d_{s}=2 \sqrt{\frac{A}{\pi}}
$$

can be calculated from the projected area $A ; d_{s}$ is the diameter of a sphere with an identical projected area. Because nonspherical ice crystals have different radiative properties in comparison to spherical water droplets, it is useful to calculate shape parameters of the imaged particle that quantify its deviation from a sphere. These are dimensionless quantities (Korolev and Isaac 2003) that are calculated by using several geometric properties extracted from the particle image. The aspect ratio of a particle

$$
\alpha=\frac{D_{w}}{D_{\max }}
$$

is the ratio between the maximum dimension $D_{\max }$ and the maximum dimension $D_{w}$ that is perpendicular with respect to $D_{\max }$ (see Fig. 4 for illustration). The roundness or area ratio

$$
\beta=\frac{4 A}{\pi D_{\max }^{2}}
$$

describes the ratio between the projected area of the particle and the area of a circle with a diameter of $D_{\max }$. Geometrically speaking, $\beta$ represents the degree of coverage of this circular area by the area $A$. The aspect ratio $\alpha$ as well as the roundness $\beta$ are confined to the interval ] $0 ; 1] \subset \mathbb{R}$. For a sphere, it is $\alpha=\beta=1$. For long and thin objects, $\alpha$ and $\beta$ are tending to zero. Some examples are shown in Fig. 4.

The quantities mentioned above are derived by applying a specially developed algorithm to the PHIPS images. This image-processing algorithm is important for the assessment of the derived particle quantities and was an essential part of the development work. Thus, the individual processing steps of this algorithm are described in detail in the following paragraphs.

Because the implemented flashlamp generates a nonuniform illumination that is different in each image, it is necessary to calculate a background image before further processing can be done. The background image is subtracted from the original image. The background image is calculated by means of a rolling ball algorithm (Sternberg 1983). In this algorithm the grayscale matrix of the original image is interpreted as a landscape with hills and valleys, where the objects of interest are represented as deep holes. A "ball" with a radius that is bigger than the largest object of interest rolls over the landscape and its trajectories are recorded. Because of its size the ball cannot penetrate the object holes and it 

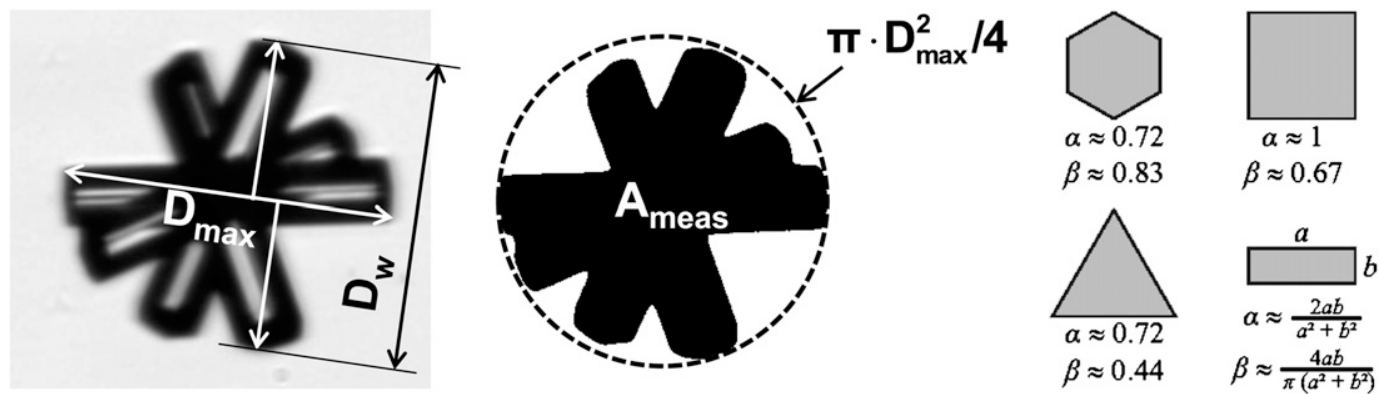

FIG. 4. Definitions of $D_{\max }$ and $D_{w}$ illustrated for the analog crystal ros220 with a projected area $A_{\text {meas. }}$ (right) Aspect ratios $\alpha$ and roundnesses $\beta$ for some selected geometrical shapes are calculated.

only follows the structure of the background that stems from the nonuniform illumination.

After having eliminated the nonuniform background the image is smoothed by means of a 5 pixel $\times 5$ pixelwide Gaussian filter. The gradients in both directions of the image are calculated by means of the Sobel operator in order to determine a region of interest (ROI) around the imaged particle. The next step is a transformation of the gradient image into a binary one with white (pixel value 1) objects on a black (pixel value 0 ) background. The threshold for the binary segmentation is calculated from the histogram $h(g)$ of the gray values $g$ in the gradient image by means of the triangle algorithm (Zack et al. 1977) that is illustrated in Fig. 5. This geometric technique works effectively if the object pixels produce only a weak peak in the histogram. By applying the Sobel operator the objects in the gradient image are embodied solely by their edges. Because the edges represent only a very small portion of the whole image, the object pixels produce only a weak peak in the grayscale histogram. The triangle algorithm starts with the identification of the most frequent gray value $g_{\max }$ and the smallest gray value $g_{\min }$ in the image. Then, a line is drawn between the two points $\left[g_{\max }, h\left(g_{\max }\right)\right]$ and $\left[g_{\min }\right.$, $\left.h\left(g_{\min }\right)\right]$. The threshold $T$ is chosen such that the distance between the drawn line and the point $[T, h(\mathrm{~T})]$ on the histogram is maximized.

The binary image that is created in this way needs further processing. It is necessary to remove objects that are touching the border of the image because they are only partly imaged and cannot be properly processed. Furthermore, it is necessary to fill background areas that are surrounded by object pixels, that is, holes in objects. Both processing routines are illustrated in Fig. 6 . The procedure starts with putting a seed frame around the border of the image. All pixels of this frame are set to a value $s$ that is not equal to the value 1 of the objects and not equal to the value 0 of the background. In the next step all of the object pixels that are connected directly (i.e., in the horizontal or vertical directions, and not diagonally) to a seed pixel are set to the value $s$. This procedure is repeated until no more object pixels are connected to a seed pixel. In the last step, all of the seed pixels are set to the background value 0 . The elimination of holes in objects works similarly. One starts again with a frame of seed pixels on the border of the image. Next, all of the background pixels that are directly connected to a seed pixel are set to the value $s$. Again, this procedure is repeated until no more background pixels are connected directly to a seed pixel. Finally, all pixels with the value $s$ are set to the background value. All other pixels are set to the object value 1 .

The processed binary image contains only compact objects that are not touching the border of the image. The last small disturbances in this binary image are eliminated by applying a morphological opening filter with a disc that has 5 pixels in diameter as the structuring element. The final binary image is multiplied with the original grayscale image to generate a masked image. In the masked image the coordinates of the steepest

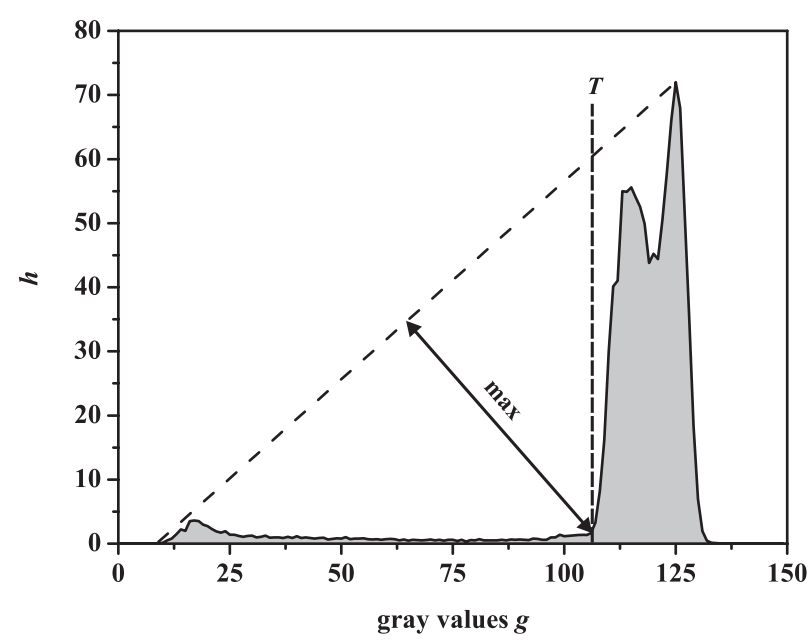

FIG. 5. Illustration of the triangle algorithm that is used to determine a threshold for the segmentation of the grayscale gradient image. 

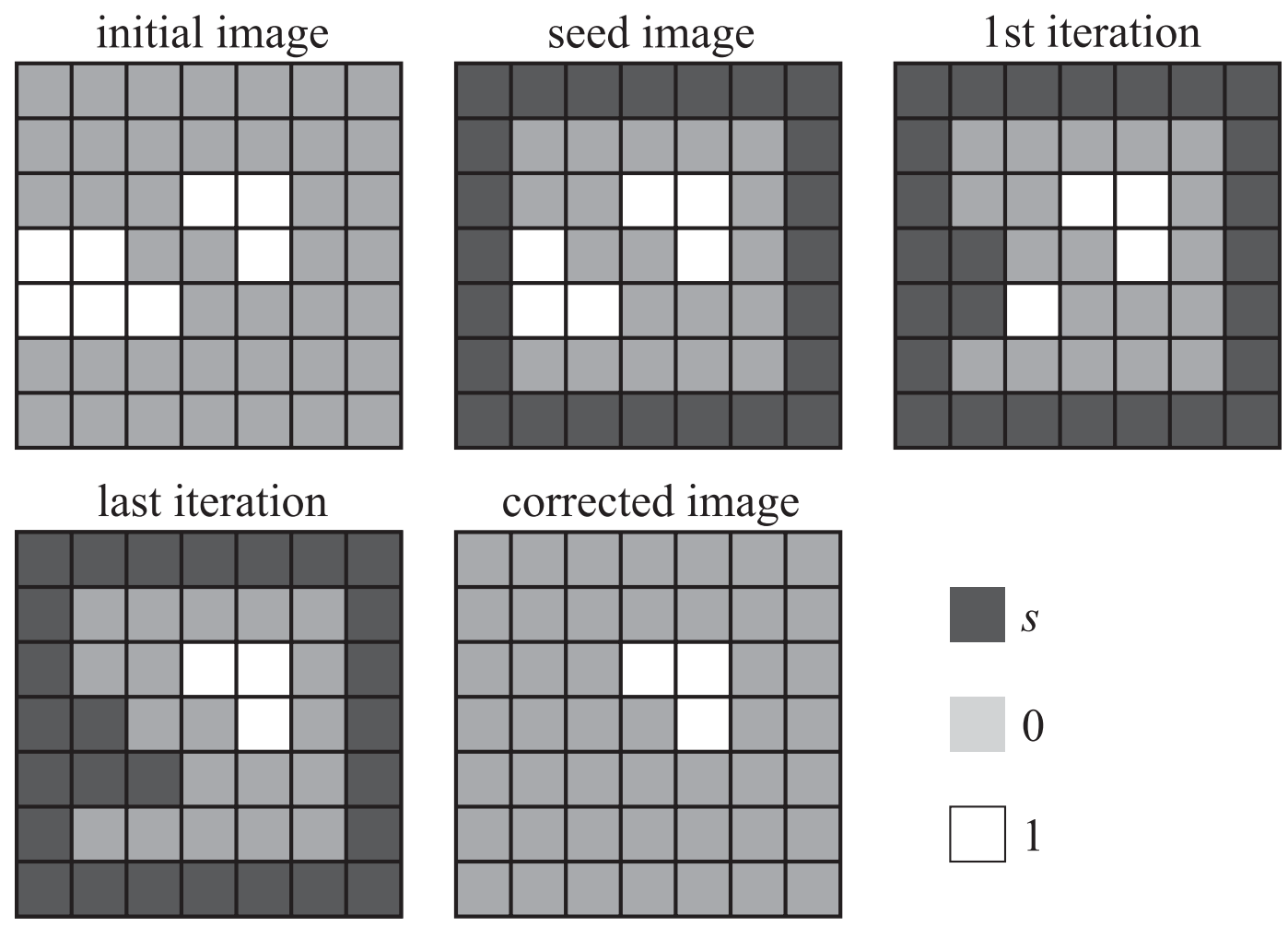

initial image
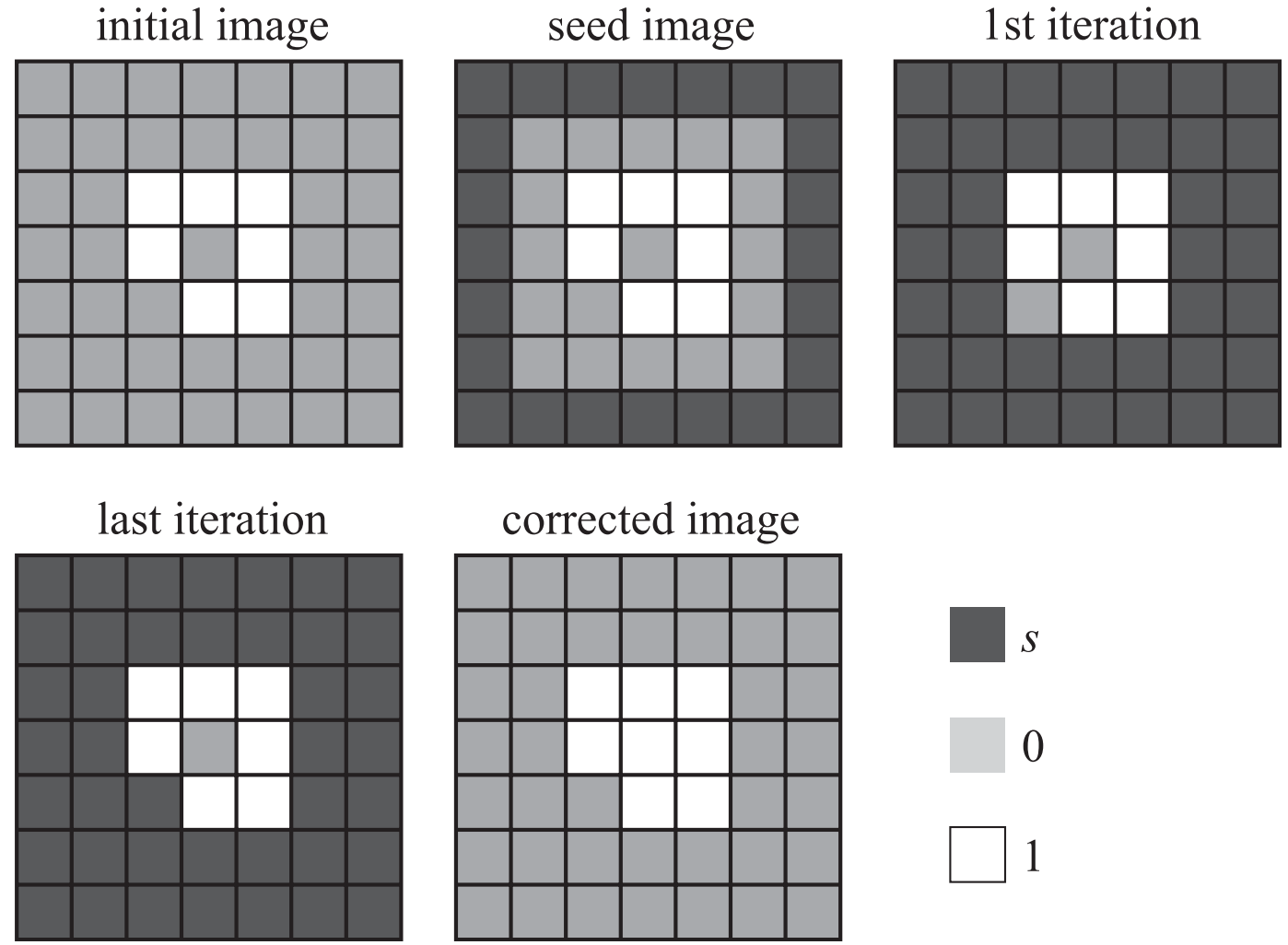

FIG. 6. Removing objects that are (top two rows) touching the border of the image and (bottom two rows) filling holes in objects. 


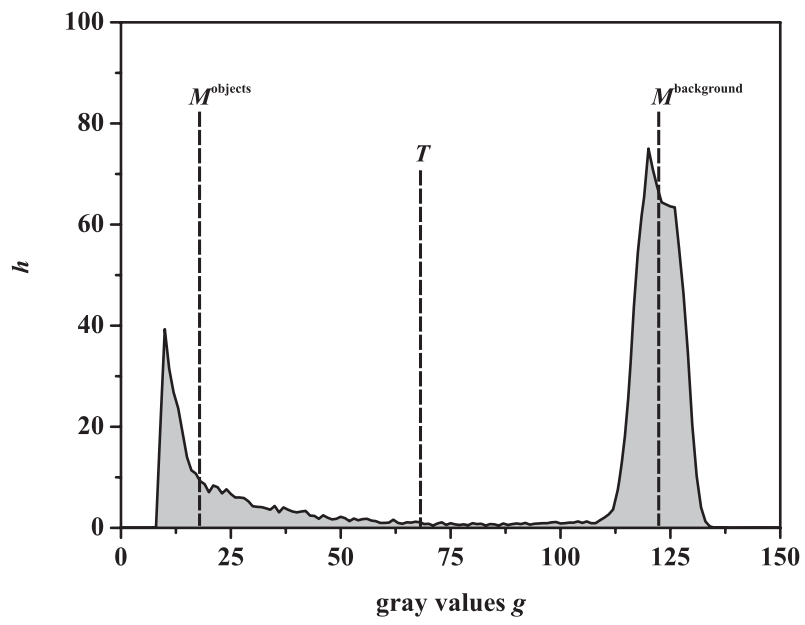

FIG. 7. Illustration of the isodata algorithm that is used to determine a threshold for the segmentation of the ROI.

gradient are determined by applying the Sobel operator, and an ROI is generated of the respective object. At this point we have to state that the whole image-processing algorithm is based on the assumption that each PHIPS image contains exactly one particle. If more than one particle is imaged by one shot, only the object that generates the steepest gradient will be processed. All of the other particles are discarded. From our observations during the AIDA experiments, we can state that over $99 \%$ of all of the acquired PHIPS images contain only one particle. Therefore, the impact of this assumption on the particle statistics can be neglected.

Both edges of the determined ROI are enlarged by a factor of 3 in order to add some padding. The ROI is then extracted from the background-subtracted original image and processed with another algorithm. The aim of this algorithm is to determine the edge points on the perimeter of the particle. The edge points are used to calculate the quantities that were described above. The procedure starts again with the transformation of the ROI into a binary image. In the ROI the object pixels produce a stronger peak in the gray value histogram than in the gradient image. Therefore, the triangle algorithm that is used to calculate the threshold for the segmentation of the gradient image is not an appropriate method to determine a threshold for the ROI. The isodata algorithm (Ridler and Calvard 1978), an iterative technique that is illustrated in Fig. 7, turned out to work very well for the calculation of a threshold for the ROI. The procedure starts with an arbitrary threshold $T_{1}$, for example, chosen as the arithmetic mean of the smallest gray value $g_{\min }$ and the biggest gray value $g_{\max }$ that is occurring in the histogram. This threshold divides the histogram into object and background pixels. In the next step the arithmetic means

$$
\begin{aligned}
M_{n}^{\text {objects }}= & \frac{\sum_{g=g_{\min }}^{T_{n}-1} g h(g)}{\sum_{g=g_{\min }}^{T_{n}-1} h(g)} \text { and } \\
M_{n}^{\text {background }}= & \frac{\sum_{g=T_{n}}^{g_{\max }} g h(g)}{\sum_{g=T_{n}}^{g_{\max }} h(g)}
\end{aligned}
$$

of the object side and the background side of the histogram are calculated. A new threshold

$$
T_{n+1}=\frac{M_{n}^{\text {objects }}+M_{n}^{\text {background }}}{2}
$$

is set and the calculation is repeated until $T_{n+1}=T_{n}$. In the resulting binary image, holes and objects at the border are removed as described above. One pixel is eroded around the entire perimeter of the objects to reduce an oversizing effect that will be discussed later in section 4 . A 3 pixel $\times 3$ pixel-wide median filter is used to eliminate salt and pepper. The shape of the particle is now completely extracted from the initial image.

For the determination of $D_{\max }$ and $D_{w}$ the perimeter of the particle is represented by a chain code (Freeman 1974). The chain code is a compact method that is used to store the shape of an object. For its determination, one starts on an abitrary pixel on the perimeter of the particle. The next pixel of the perimeter is then defined by a direction. There are eight possible directions that can be encoded by the numbers $1-8$, for instance. For the determination of $D_{\max }$ by means of the chain code an algorithm is used that steps through the chain and simply calculates all of the distances between all of the possible pairs of perimeter pixels. To measure $D_{w}$, the two pixels associated with $D_{\max }$ are used to divide the perimeter into two parts. Stepping from pixel to pixel through the two parts of the perimeter, and subsequently calculating the distance from the axis of $D_{\max }$ on both of its sides, delivers $D_{w}$. The chain code is also used for the calculation of the projected area. The procedure is too lengthy to be described here. Because it does not have any advantage to simple pixel counting, but some saving of time, the interested reader may refer to Jähne (2002) for a detailed description.

\section{Results}

\section{a. Characterization of the imaging properties}

To get a quantitative description of the depth of field, the resolving power, and the imaging properties of the 
TABLE 1. Crystal name, projected area $A$, and sphere-equivalent diameter $d_{s, \text { ref }}$ of the imaged ice analogs.

\begin{tabular}{lrr}
\hline \hline Crystal name & $A\left(\mu \mathrm{m}^{2}\right)$ & $d_{s, \text { ref }}(\mu \mathrm{m})$ \\
\hline col10 & 42 & 7.3 \\
cros12 & 70 & 9.4 \\
col15 & 90 & 10.7 \\
col20 & 150 & 13.8 \\
col26 & 198 & 15.9 \\
cros20 & 234 & 17.3 \\
col53 & 820 & 32.3 \\
ros41 & 953 & 34.8 \\
col84 & 2029 & 50.8 \\
gros56 & 2143 & 52.2 \\
ros80 & 3130 & 63.1 \\
ros150 & 12300 & 125.1 \\
ros160 & 16200 & 143.6 \\
ros220 & 27700 & 187.8 \\
\hline
\end{tabular}

microscope unit, ice analogs (Ulanowski et al. 2003) were used as realistic test objects. These are tiny (several microns) crystals made of sodium hexafluorosilicate $\left(\mathrm{Na}_{2} \mathrm{SiF}_{6}\right)$. Like ice, these crystals exhibit the shape of hexagonal columns and plates as well as complex combinations (e.g., rosettes) of these two basic structures. Their refractive index is similar to that of ice $(\sim 1.31)$. Unlike ice, $\mathrm{Na}_{2} \mathrm{SiF}_{6}$ is completely stable at room temperature. Test measurements can thus be performed without any complex cooling. Each analog particle imaged by PHIPS was measured thoroughly in advance under the same particle orientation by means of high-resolution microscopy in order to deduce the projected area $A$ and the sphere-equivalent diameter $d_{s, \text { ref }}$ with high accuracy. The resulting parameters are listed in Table 1 for the employed analogs. Figure 8 shows the corresponding PHIPS images together with reference images of the rosettes "ros220" and "ros160" from high-resolution microscopy. Single facets could be resolved down to a crystal size of $14 \mu \mathrm{m}$. In this context the crystal named ros160 should be mentioned. This crystal has rough facets, as confirmed by high-resolution microscopy, and this roughness is clearly resolved by the imaging system of PHIPS. Rough ice particles may be dominant in some mixed-phase and cirrus clouds (Ulanowski et al. 2010) and may have a significant impact on the radiative properties of these clouds, because the scattering phase function of rough ice analogs differs clearly from that of crystals with smooth surfaces (Ulanowski et al. 2006).

Each ice analog is fixed on the surface of a microscope slide by adhesion forces. To quantify the influence of the defocusing on the image quality, the slide with the particle was moved stepwise along the optical axis through the depth of field of the microscope system by means of a micrometer-driven translation stage. At each $50-\mu \mathrm{m}$ step an image was captured and processed, and the projected area and the sphere-equivalent diameter were calculated. In the upper plots of Fig. 9, the ratio between the sphere-equivalent diameter $d_{s}$ determined by PHIPS

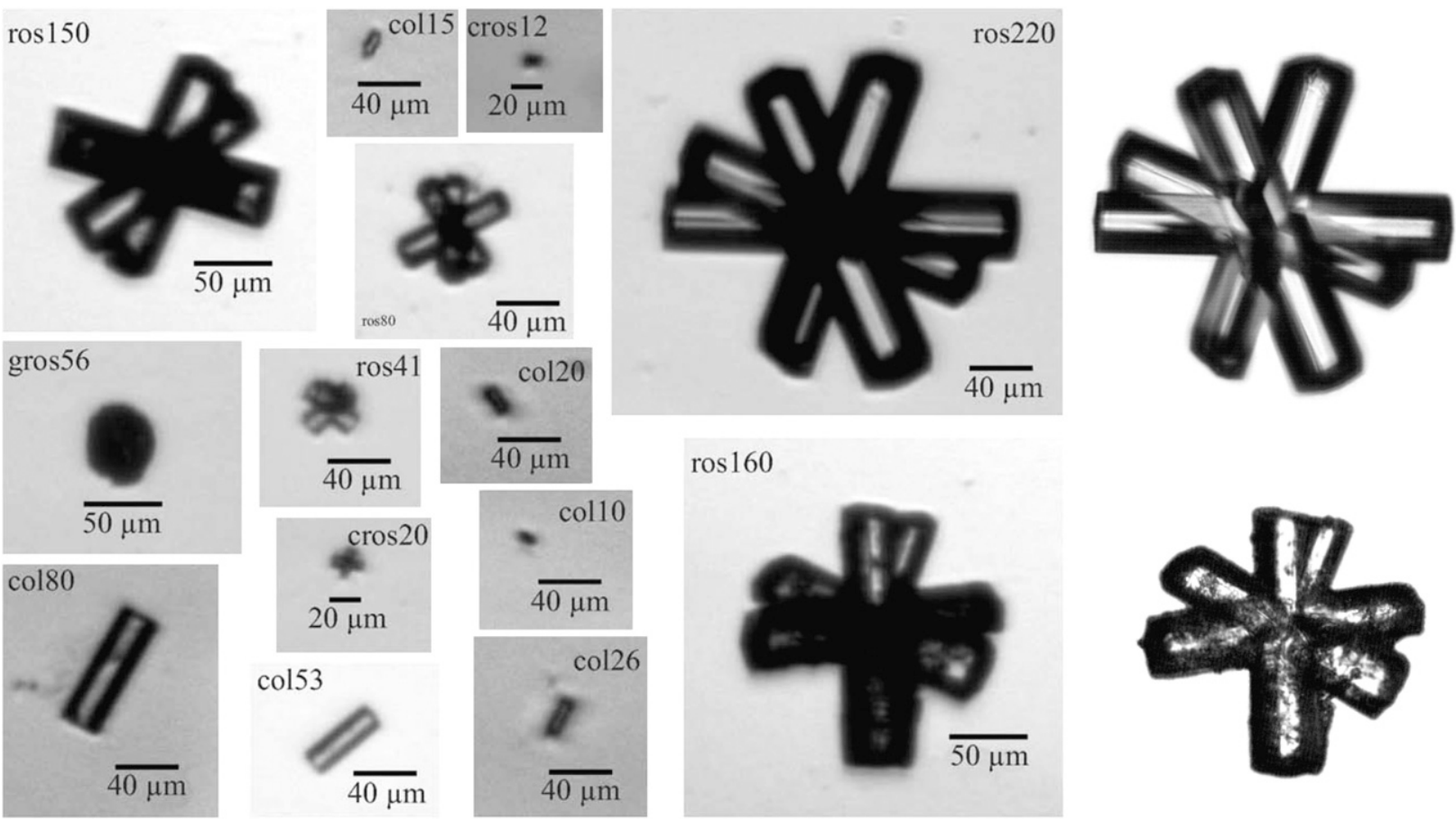

FIG. 8. (left) Ice analogs imaged by PHIPS. (right) Images from high-resolution microscopy of the smooth crystal ros 220 and ros 160 with rough facets are depicted to illustrate the image quality of PHIPS. 

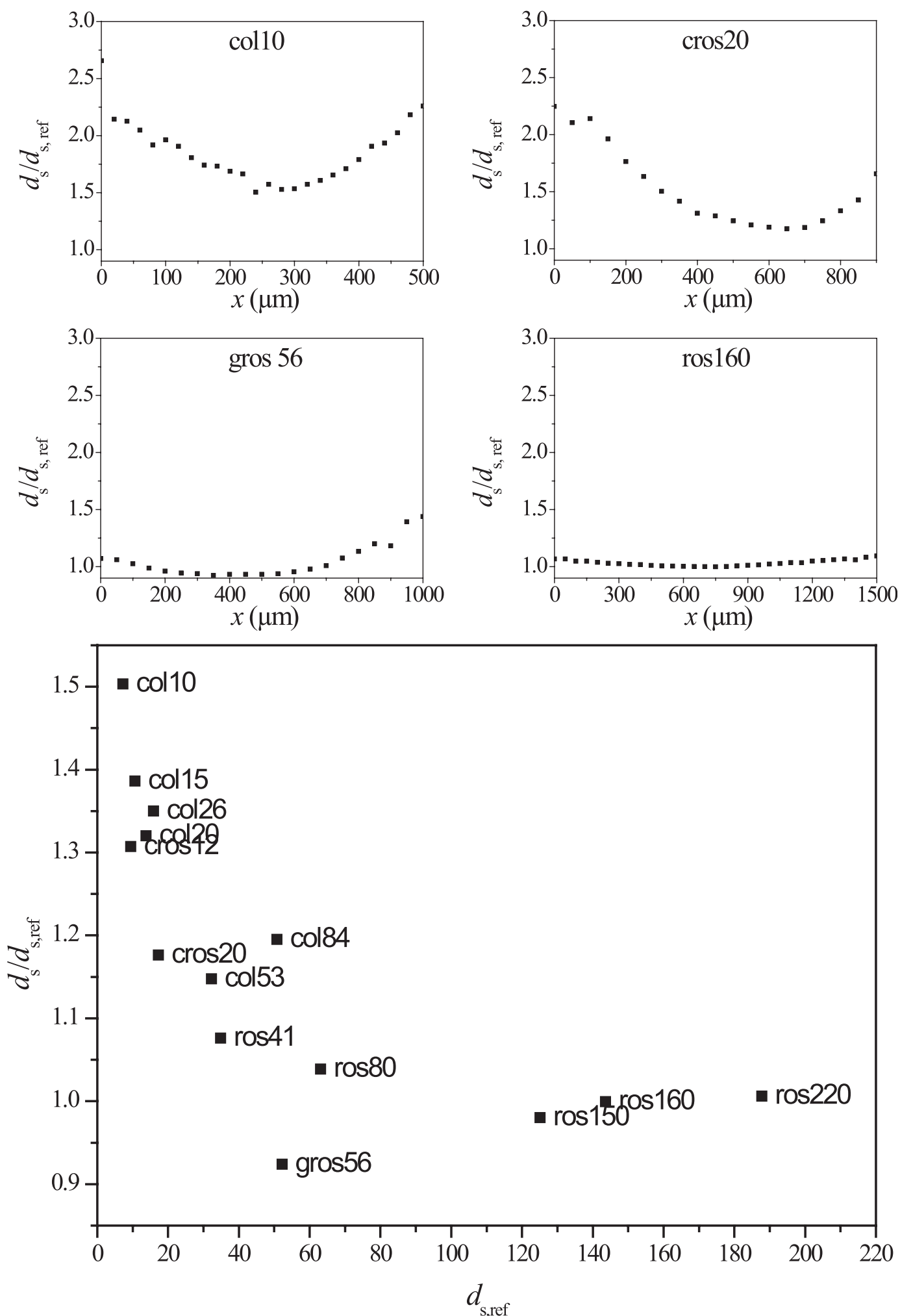

FIG. 9. (top) Effect of defocusing on the measured value for the sphere-equivalent diameter $d_{s}$ of the imaged ice analogs, which was determined by the digital image processing and analysis procedure described in section 3 . The sphere-equivalent diameter of each ice analog is normalized to the "true" value $d_{s, \text { ref }}$, which has been determined by means of high-resolution microscopy in advance to deduce the oversizing ratio. (bottom) Size dependence of the oversizing ratio for in focus particles. 
and its independently measured reference value $d_{s, \text { ref }}$ is plotted as a function of the object depth $x$ for a selection of analog particles. From these plots, the usable depth of sharpness of the imaging optics can be estimated. We typically find a region of $200-300-\mu \mathrm{m}$ depth around the focal plane where the ratio $d_{s} / d_{s, \text { ref }}$ does not change too much. Because only those particles are imaged by PHIPS that are confined to a range of $\pm 50 \mu \mathrm{m}$ of the focal plane, as explained by the insert in Fig. 1, all ice crystals larger than the ice analog "gros56" listed in Table 1 are expected to be imaged by PHIPS without significant overestimation of $d_{s}$.

For the analogs with smaller sphere-equivalent diameters, there is an increasing overestimation with decreasing particle size, up to a factor of 1.5 for the smallest analog particle "col10" (see lower plot in Fig. 9). This is most likely due to the fact that there is an increasing impact of the resolving power of the microscope unit with decreasing particle size. A further impact might stem from the image analysis procedure itself, which could have problems with the impaired resolution and contrast in case of images of small particles. In principle, a correction function could be deduced from the presented analog measurements, but the current dataset is insufficient and, thus, the determination of a correction function will be the subject of forthcoming work. However, we could show in an expansion-cooling experiment at AIDA, where small columnar ice particles have been nucleated and grown to sizes well below $20 \mu \mathrm{m}$ at a temperature around $-45^{\circ} \mathrm{C}$, that the particle oversizing by PHIPS can be corrected on the basis of the analog measurements. Figure 10 shows the comparison of the mean equal volume sphere diameter $d_{v}$ of the ice particles as retrieved from Fourier transform infrared (FTIR) spectroscopy with the result of the PHIPS single particle analysis. Details of this experiment and the FTIR retrieval method can be found in Wagner et al. (2009). For this comparison the sphere-equivalent diameter deduced by PHIPS was first downscaled by a factor of 1.6 in order to correct for the small particle oversizing and was subsequently converted into an equal volume sphere diameter assuming randomly oriented ice cylinders of aspect ratio one, which gave the best FTIR retrieval.

The PHIPS data can be compared with the oversizing of particles imaged by the CPI instrument that has been investigated by Connolly et al. (2007). They also used ice analogs of different sizes and habits. Although both instruments are not strictly comparable because PHIPS uses an incoherent visible flashlight, while the laser diode of the CPI emits coherent infrared pulses that give rise to significant diffraction fringes, both studies yielded comparable depth-of-field dependencies of particle oversizing.

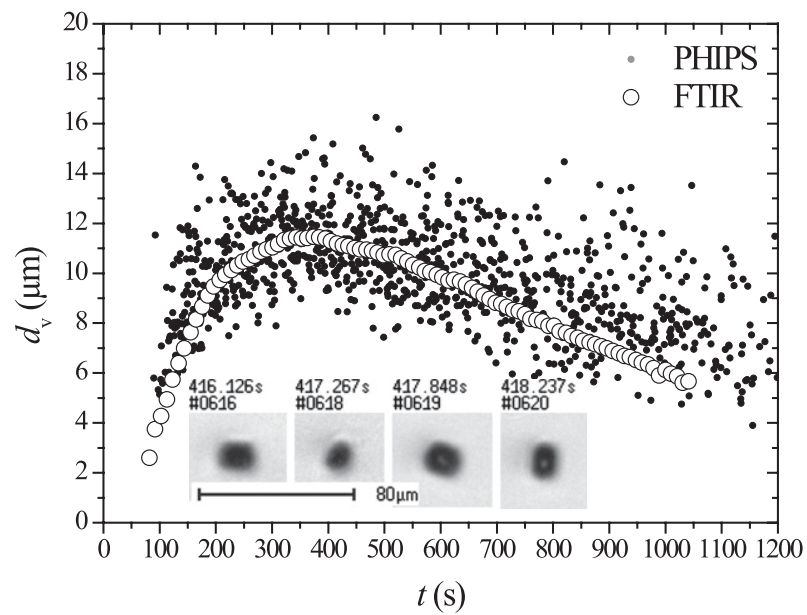

FIG. 10. Comparison of the mean equal volume sphere diameter $d_{v}$ deduced from the PHIPS images (dots) and retrieved from FTIR extinction spectroscopy (open circles). See text for details.

The interference fringes in the CPI images made it possible to develop an algorithm that is capable of correcting the oversizing of out-of-focus particles. In view of the superior imaging properties of PHIPS we are confident that a correction algorithm that does not rely on diffraction fringes can be developed in the near future. This would allow us to improve ice particle statistics in AIDA experiments by increasing the useful optical depth of PHIPS.

\section{b. Temperature dependence of ice crystal habits in AIDA experiments}

It has been known since the 1930s that the habit of ice crystals is dependent on both temperature and ice supersaturation prevailing during crystal growth [see e.g., Libbrecht (2005) for a recent review]. The dependencies of the crystal morphology on the temperature and ice supersaturation have been investigated in various laboratory and field studies and are usually illustrated in a so-called ice crystal morphology diagram. The various diagrams (e.g., Kobayashi 1961; Bailey and Hallet 2009) show congruent features in the temperature range between $0^{\circ}$ and $-20^{\circ} \mathrm{C}$. Thus, we refer to all of these diagrams by using the term "morphology diagram" in the following.

In this section, comprehensive datasets from pure and mixed-ice cloud observations at $256 \mathrm{~K}\left(-17^{\circ} \mathrm{C}\right)$, between 267 and $268 \mathrm{~K}\left(-6^{\circ}\right.$ and $\left.-5^{\circ} \mathrm{C}\right)$, and between 258.5 and $259.5 \mathrm{~K}\left(-14.5^{\circ}\right.$ and $\left.-13.5^{\circ} \mathrm{C}\right)$ are presented from three selected AIDA chamber experiments. They covered a temperature range in which a transition from columns to plates and to more complex morphologies at elevated ice saturation ratios during growth are expected from the morphology diagram. The ice seed generator 


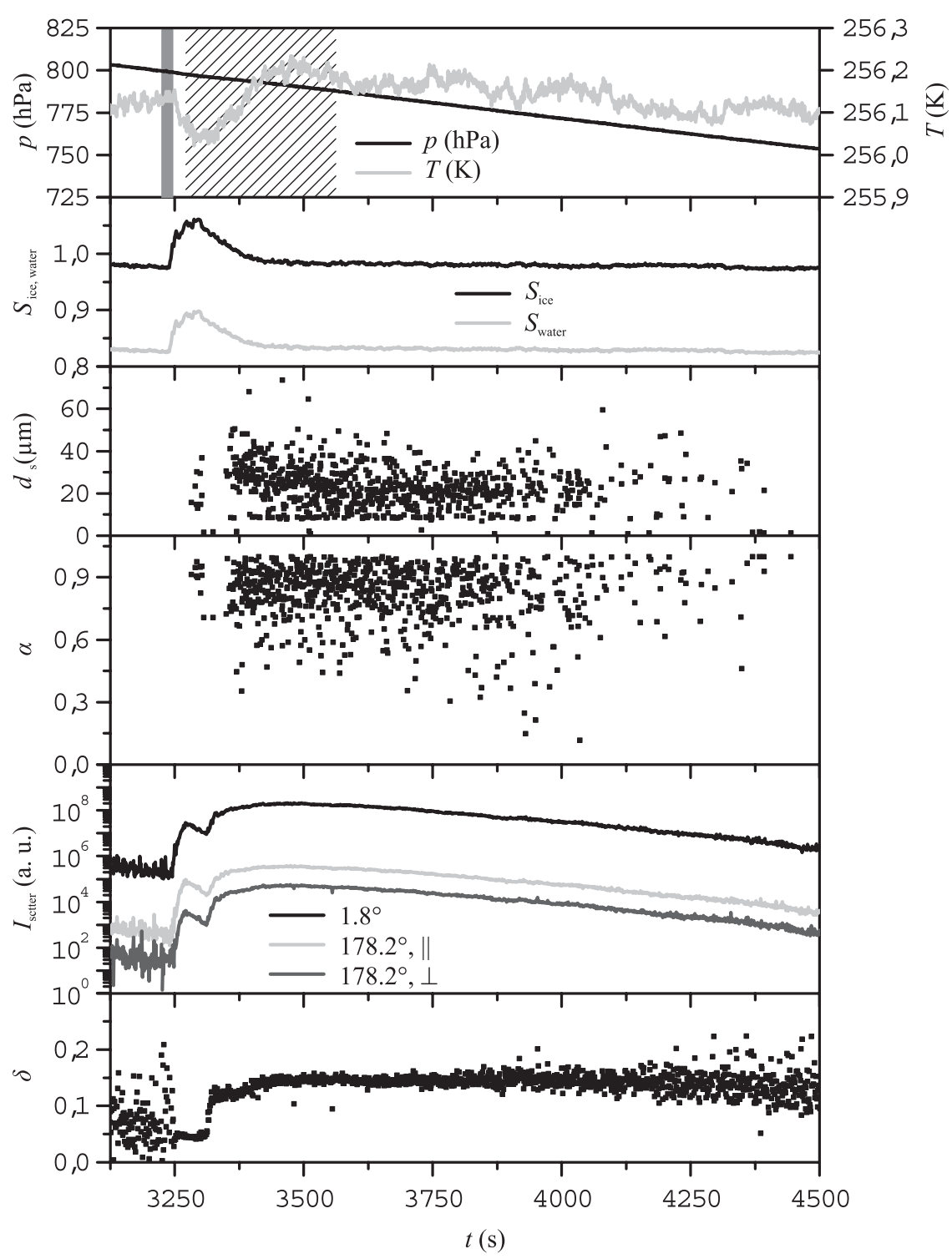

FIG. 11. The chronology of the AIDA experiment IN08_40 that was performed at $256 \mathrm{~K}$. (from top to bottom) The pressure $p$, gas temperature $T$, saturation ratio with respect to ice $S_{\text {ice }}$ and water $S_{\text {water }}$, sphere-equivalent diameter $d_{s}$, aspect ratio $\alpha$, forward- and backscattering signal $I_{\text {scatter }}$, and depolarization $\delta$ are plotted as a function of the time $t$ elapsed during the experiment. In the topmost diagram, the periods when water droplets (gray) and ice seeds (hatched) had been injected are marked.

described in section 2 was used to generate an initial ice particle population inside the AIDA chamber. To maintain supersaturated conditions needed for crystal growth, the chamber was slowly evacuated during the experiments. Thus, the pressure inside the chamber was slowly lowered, resulting in a slow decrease of the temperature. In this way, a temperature slightly below the frost point could be maintained. If necessary, the available amount of water vapor could be raised by spraying in supercooled water droplets.

\section{1) ICE PARTICLE GROWTH AT $256 \mathrm{~K}\left(-17^{\circ} \mathrm{C}\right)$}

The chronology of the AIDA experiment IN08_40 that was carried out at $256 \mathrm{~K}$ is shown in Fig. 11. At this temperature mainly hexagonal plates should grow. At the beginning of the experiment the chamber had been slowly evacuated until ice saturation was reached. For a further increase of the ice saturation ratio, supercooled water droplets were injected (indicated by the gray area in the topmost diagram in Fig. 11). The droplets 


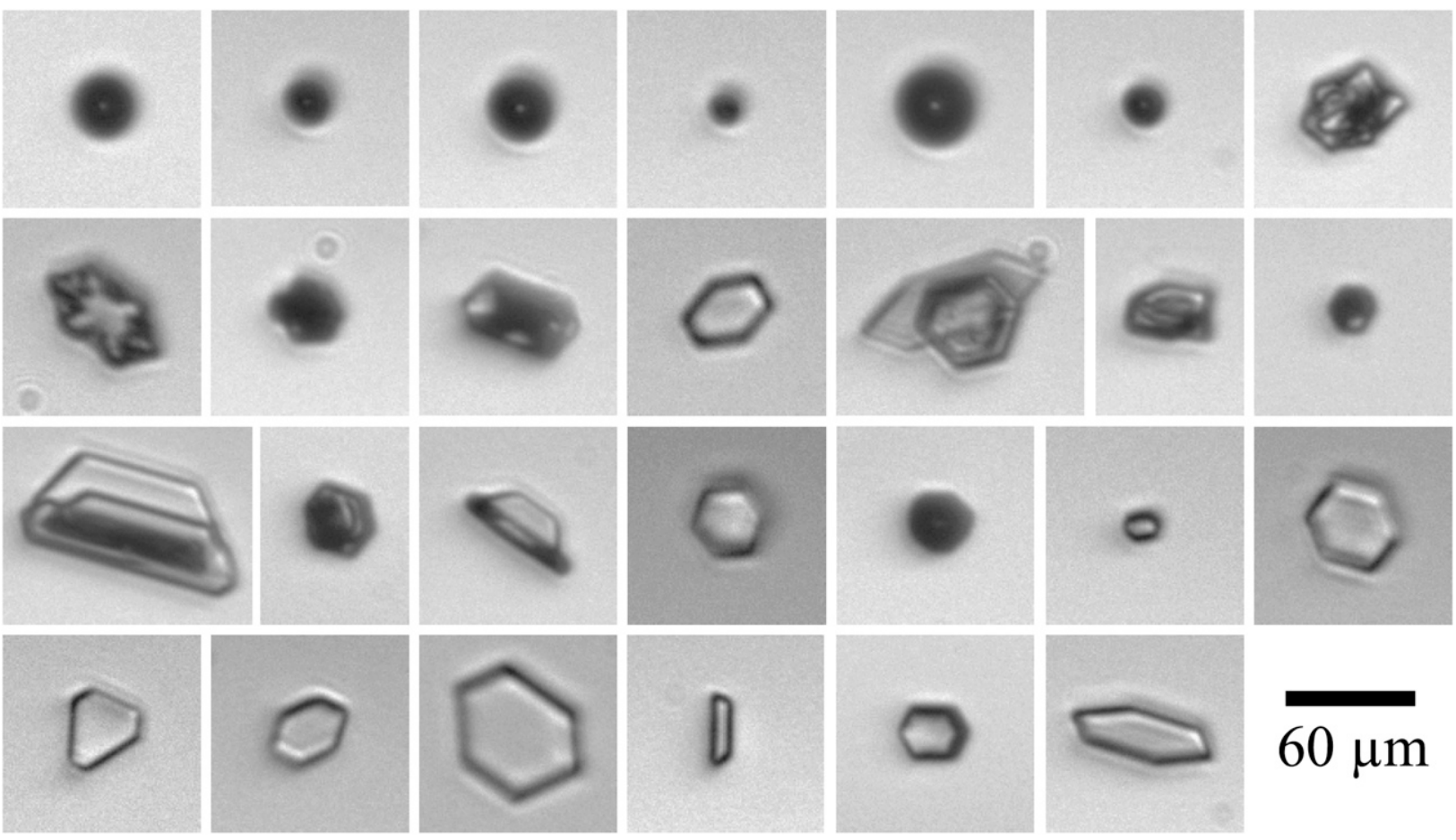

FIG. 12. A subjective selection of crystals that had been imaged by PHIPS during the experiment IN08_40 performed in the AIDA chamber at $256 \mathrm{~K}$. (from top left to bottom right) The images are tabulated in their chronological order. (top) One can recognize the injected water droplets. After the water droplets, two crystals were captured that have been grown at a still-high ice saturation ratio. These particles can be classified as aggregates of plates, side planes, and skeleton plates.

evaporated inside the chamber, which led to a short temperature decrease resulting from the absorption of latent heat. The presence of droplets in the chamber is reflected by the strong increase of the scattering signals $I_{\text {scatter }}$ and the low, near-background value $(\sim 0.04)$ of the depolarization ratio $\delta$ of the laser light that is backscattered from the center of the chamber. The injection of the ice seed particles (indicated by the hatched area in the topmost diagram in Fig. 11) was started just before the ice saturation ratio reached its maximum. SIMONE ranked first in detecting the formation of an ice cloud, because it measures scattering intensity and depolarization, parameters that are significantly affected by even the smallest ice crystals. As soon as the crystals had grown into the size range that can be detected by the Welas counters, the ice particle-imaging instruments were turned on. The depolarization ratio $\delta$ increased from its background value during the droplet phase to values between 0.10 and 0.15 for the ice particle phase, which is comparable to the results of a recent AIDA ice cloud study for a cloud period composed of thick plates and aggregates of plates (Amsler et al. 2009). Seed ice particles were added for $\sim 5 \mathrm{~min}$. After switching off the seed generator the ice cloud was sustained for more than 15 min by carefully controlling the pumping speed, as described above. In the third and fourth panels of Fig. 11, the sphere-equivalent diameters $d_{s}$ and the aspect ratios $\alpha$, which were obtained from all of the evaluable images captured during the experiment, are plotted against the experiment time. Each dot in the scatterplots represents one imaged ice crystal. The crystals were grown to a maximum sphere-equivalent diameter of $\sim 80 \mu \mathrm{m}$. The aspect ratio can be calculated to values between 0.5 and 1.0, which corresponds to the crystal shapes pictured in Fig. 12. The fact that most of the plates imaged by PHIPS are orientated with their basal planes parallel to the velocity vector (which points horizontally from left to right in all particle images) is due to the parabolic velocity profile inside the sampling tube that persists up to the imaging region of PHIPS. The observed shape-dependent orientation of ice crystals that are imaged by PHIPS is a disadvantage of the instrument in its present configuration. We intend to eliminate the problem in the future by following the concept of the CPI instrument where particle orientation is randomized by establishing turbulent flow conditions in the sampling tube. However, other unwanted side effects of turbulent flow conditions, like a reduction of the sampling probability, must be carefully investigated.

The aspect ratio $\alpha \approx 0.72$ for hexagonal plates that is quoted in Fig. 4 is only valid if their basal planes are 
oriented exactly parallel to the object plane of the microscope. In this orientation the projected area of the crystal, and thus $D_{w}$, is maximal. All other orientations result in smaller aspect ratios. In the experiment at $256 \mathrm{~K}$ where ice plates were by far the prevailing crystal shape, aspect ratios as low as $\sim 0.2$ were observed. This is more typical of an elongated column, but can also result from the projection of a thin plate with its basal facet preferentially orientated perpendicular with respect to the object plane of the microscope, resulting in a minimal projected area. When the hexagonal axis of the ice plate is orientated exactly parallel to the object plane, the aspect ratio is minimized, and the perpendicular diameter $D_{w}$ corresponds exactly to the thickness of the plate.

The problem of incorrect ice crystal classification resulting from orientation ambiguity can be reduced by flashing and imaging ice crystals simultaneously under different angles. An improved version of the PHIPS instrument is presently being tested, which includes optics for the dual imaging of ice crystals and simultaneous measurements of their scattering function at very high angular resolution $\left(1^{\circ}\right.$ in the forward direction).

\section{2) ICE PARTICLE GROWTH BETWEEN 267 AND $268 \mathrm{~K}\left(-6^{\circ}\right.$ AND $\left.-5^{\circ} \mathrm{C}\right)$}

The chronology of another AIDA experiment (IN08_42) dealing with crystal shapes is depicted in Fig. 13. The experimental procedure was similar to that in the previous section. According to the ice crystal morphology diagram, mainly hexagonal columns should be generated at temperatures between 267 and $268 \mathrm{~K}$ and low supersaturations with respect to ice. The injection of ice seeds (indicated by the hatched area in the topmost panel of Fig. 13) was started immediately at the beginning of the experiment. Because ice saturation was not reached during the initial phase of the experiment, the seed crystals evaporated immediately during the first $\sim 4$ min. While continuing the injection of seed ice particles, the relative humidity over ice was raised by increasing the pumping speed. As soon as ice saturation was reached, PHIPS and SIMONE started detecting ice crystals. A weak undersaturation with respect to ice between $\sim 400$ and $\sim 500 \mathrm{~s}$ resulted from a slight reduction of the pumping speed. To prevent the ice cloud from evaporating, the pumping speed was increased appropriately. In this way, ice-saturated conditions were maintained for $\sim 20 \mathrm{~min}$. During the first period of injecting ice seeds, columnar crystals were observed, as expected on the basis of the ice morphology diagram. Because of the columnar particle shape, the depolarization ratio $\delta$ reached values of around 0.2 , which are slightly above the values found for the platelike crystals in the previous experiment at $256 \mathrm{~K}$. The difference in the depolarization ratios is in accordance with the results of earlier laboratory investigations performed at the Colorado State University cloud chamber [see Sassen (1991) for a summary]. After the injection of seed ice was stopped, the cloud could be observed for another $10 \mathrm{~min}$. After the cloud had evaporated, seed ice was injected once more for $4 \mathrm{~min}$ at an appropriate pumping speed to create a second ice cloud. It persisted for $\sim 10 \mathrm{~min}$. When the cloud was about to evaporate, water was sprayed into the chamber for $10 \mathrm{~s}$. The sudden enhancement of the ice supersaturation resulting from the evaporating water droplets gave rise to the growth of needlelike columns. At least part of the columns that are shown in Fig. 14 were definitely hollow. The transient presence of supercooled water droplets in the ice cloud is also reflected by a sudden dip in the depolarization signal, which recovered as the droplets evaporated.

The aspect ratios calculated for the ice crystals imaged in this experiment reached values as low as $\alpha<0.1$, which are typical for long and thin objects. In this experiment we operated a CPI in parallel to our PHIPS. Both instruments were attached to the bottom of the AIDA chamber, sampling hydrometeors through vertical tubes of the same length and diameter. However, the CPI was operated at a higher sampling speed to create turbulent flow conditions in the tube in order to randomize the orientation of the sampled ice crystals in the field of view of the CPI while the PHIPS instrument sampled under the laminar flow conditions. The impact of this difference on particle orientation is illustrated in Fig. 14, which compares PHIPS and CPI images of selected columnar and needlelike ice crystals from this experiment in the upper and lower panels: the columns, in particular the very long ones, are strongly aligned along the gas flow direction in the PHIPS images, while little or no orientation is evidenced in the CPI images. Figure 14 also emphasizes the superior imaging properties of PHIPS, which is partially due to the somewhat higher-resolving power of the microscope-diode array combination, but, in particular, to the absence of interference fringes, which blur the CPI images as a consequence of the coherent illumination.

The 2-min time averages of the sphere-equivalent diameters deduced from the PHIPS and the CPI images are compared in Fig. 15. Although the ice crystals are strongly horizontally orientated in the PHIPS images, and while no preferential orientation was found in the CPI images, the two datasets agree within their combined error limits in terms of the time evolution and absolute values of their sphere-equivalent diameters $d_{s}$. 


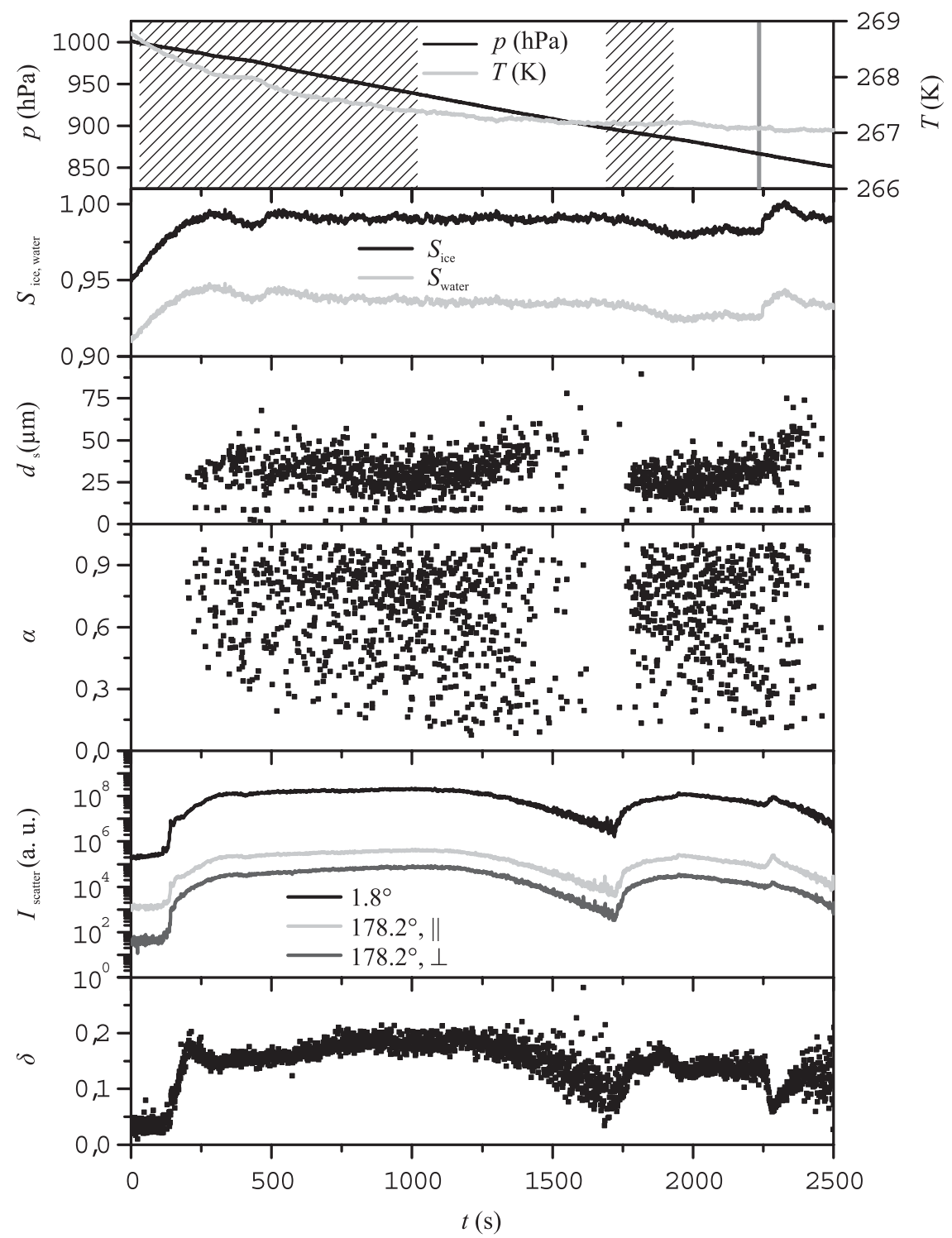

FIG. 13. The chronology of the AIDA experiment IN08_42 that was performed at temperatures between 267 and $268 \mathrm{~K}$. (from top to bottom) The pressure $p$, gas temperature $T$, saturation ratio with respect to ice $S_{\text {ice }}$ and water $S_{\text {water }}$, sphere-equivalent diameter $d_{s}$, aspect ratio $\alpha$, forward- and backscattering signal $I_{\text {scatter }}$, and depolarization $\delta$ are plotted as a function of the time $t$ elapsed during the experiment. (top) The periods when ice seeds had been injected are marked (hatched). At $t \approx 2250 \mathrm{~s}$ the interval shaded in gray marks the brief injection of water droplets.

\section{3) ICE PARTICLE GROWTH BETWEEN 258.5 AND $259.5 \mathrm{~K}\left(-14.5^{\circ}\right.$ AND $\left.-13.5^{\circ} \mathrm{C}\right)$}

Figure 16 shows the chronology of the last experiment (IN09_33) that will be discussed in this paper, where an ice cloud could be observed in the temperature range between 258.5 and $259.5 \mathrm{~K}$. In this range the formation of ice plates is expected according to the ice morphology diagram. At the beginning of the experiment, the AIDA chamber was slowly evacuated until ice saturation was reached. Then, seed ice injection was started and the growth of the ice particles was observed with PHIPS, two Welas sensors, and the SIMONE device. During the period of seed ice addition that lasted for $\sim 18.5 \mathrm{~min}$, crystals with a sphere-equivalent diameter $d_{s}$ of up to $\sim 70 \mu \mathrm{m}$ were observed. Immediately after the seed ice addition was stopped, water droplets were sprayed into the chamber for about 3.5 min during which time ice 


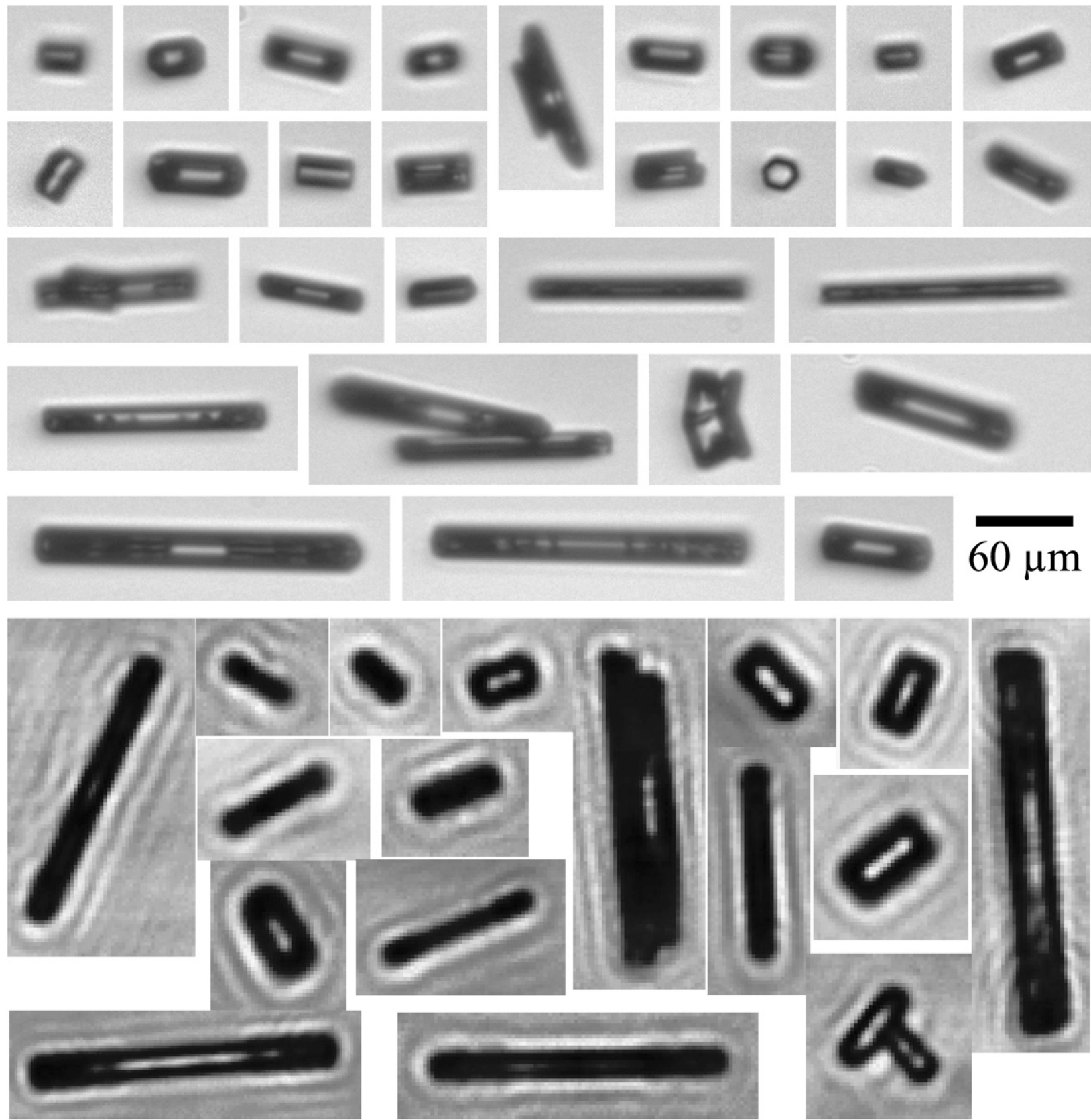

FIG. 14. A subjective selection of crystals that had been imaged by (top) PHIPS and (bottom) CPI during experiment IN08_42. The sixth image in the second row displays a regular hexagon. Although this crystal has the appearance of a plate, it is most likely the basal facet of a column. (from top to bottom) The PHIPS images are tabulated in their chronological order. The very large and long columns were observed after spraying water droplets into the ice cloud.

saturation was strongly exceeded. This resulted in the evolution of very large complex crystals with sphereequivalent diameters $d_{s}$ of up to $\sim 200 \mu \mathrm{m}$, almost the size of snowflakes. A selection of PHIPS images from experiment IN09_33 is shown in Fig. 17. The images show platelike habits of the ice crystals with different degrees of complexity: aggregates of pristine crystals were observed at low supersaturations and large complex crystals were observed after the droplets have been sprayed into the ice cloud. Most of the large crystals can be classified as sector plates and stellars, which is in agreement with the expectations from the morphology diagram for the ice crystal growth in the temperature range between $-10^{\circ}$ and $-20^{\circ} \mathrm{C}$ and near water saturation. A closer look at 

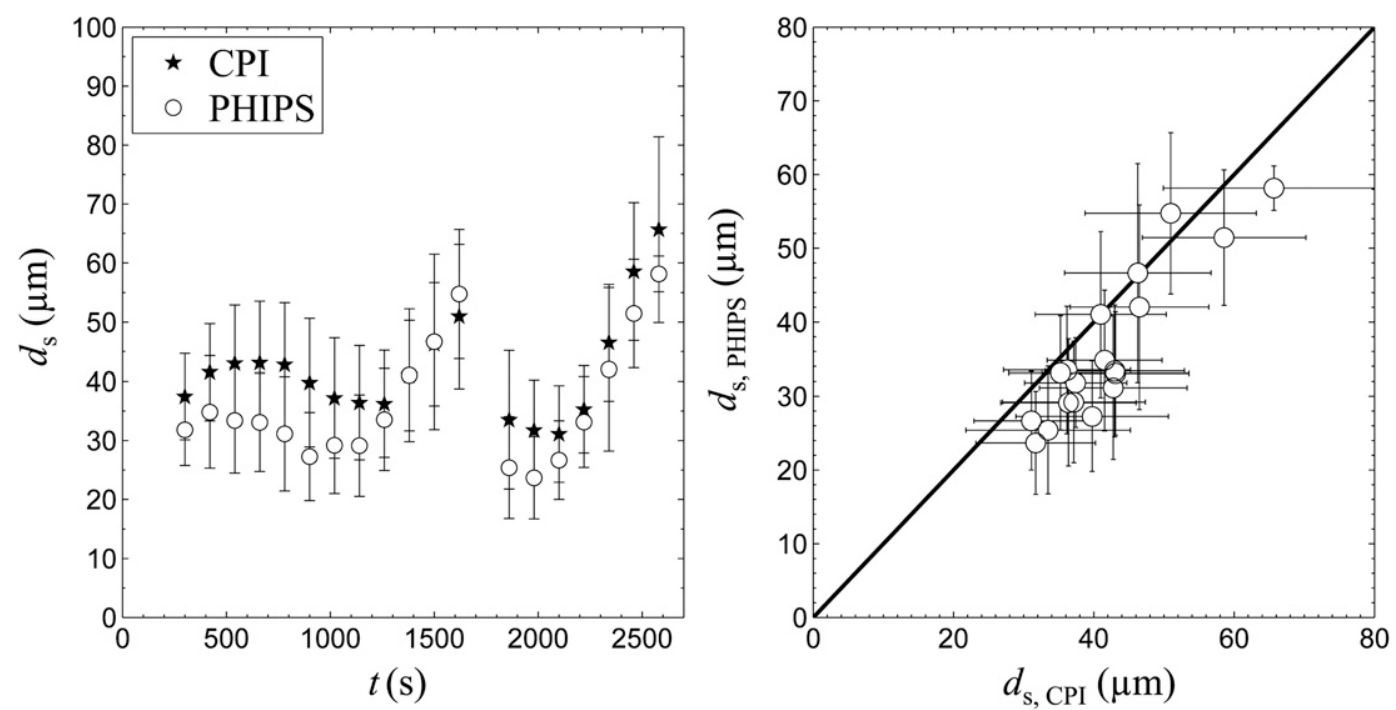

FIG. 15. Comparison of the sphere-equivalent diameter $d_{s}$ deduced from the CPI and PHIPS images of experiment IN08_42.

the PHIPS images of the sector plates occasionally reveals first signs of dentritic branching. Given that the maximum value of $S_{\text {ice }}$ shown in the second upper panel of Fig. 16 was $\sim 1.05$, dendritic structures of the ice crystals seems to disagree with the morphology diagram, which predicts that very high ice supersaturations of about $\sim 1.18$ would be required to produce such structures. Note, however, that the local ice supersaturation close to the droplet injector (where ice crystal growth is expected to be fastest) was most likely much higher than the volume averaged $S_{\text {ice }}$, which is shown in Fig. 16. On the other hand, it certainly did not exceed (and probably remained below) $S_{\text {ice }}$, corresponding to water saturation in the presence of supercooled droplets at the prevailing temperature. This contrasts with the morphology diagram, which requires the exceedance of the water saturation line to produce dendrites. The same holds true for the evolution of needlelike ice crystals in the previous experiment (see Fig. 14), which evolved near $267 \mathrm{~K}$ while approaching (but not exceeding) water-saturated conditions in the vicinity of the droplet injector.

In conclusion, the morphology diagram that is widely accepted in the temperature range between $0^{\circ}$ and $-20^{\circ} \mathrm{C}$ (Bailey and Hallet 2009) was found to correctly predict the dominant ice crystal shapes as a function of the temperature, while it overpredicts the ice supersaturations that are needed to generate more complex structures such as hollow columns, very long needles, and dendrites. It should also be noted that it was not possible to find experimental conditions that yield only ice columns, or only ice plates, or other regular structures like those shown in Figs. 12, 14, and 17. A large fraction of irregularly shaped particles was additionally observed. Some of them could be shown, by virtue of the excellent imaging properties of PHIPS, to be aggregates of more regular crystals that agreed with the $T$ dependencies of the morphology diagram.

\section{Summary and outlook}

The PHIPS instrument was developed to image airborne ice crystals at an optical resolving power of $2 \mu \mathrm{m}$. The first version of the instrument was constructed to examine crystal shapes in artificial ice clouds at the cloud simulation chamber AIDA of the Karlsruhe Institute of Technology. Because of the incoherent bright field illumination with ultrashort pulses of incoherent white light, the instrument captures shadow images that are free of diffraction fringes and motion blurring. The optical properties of PHIPS, in particular, the depth of sharpness of the microscope, have been characterized by means of ice analog crystals, as detailed in section $4 a$. It was found that the sphere-equivalent diameter $d_{s}$ of very small ice crystals may be overestimated by up to $50 \%$. This effect vanishes for larger ice crystals.

The new instrument was successfully deployed in a series of experiments at the AIDA chamber over a wide range of temperatures above the homogeneous frost point of water droplets. The predictions of the ice morphology diagram reported by different authors were confirmed in terms of the occurrence temperature of columnar versus platelike ice crystals. However, it could be shown that the ice supersaturations needed to generate very long needlelike ice columns or dendritic 


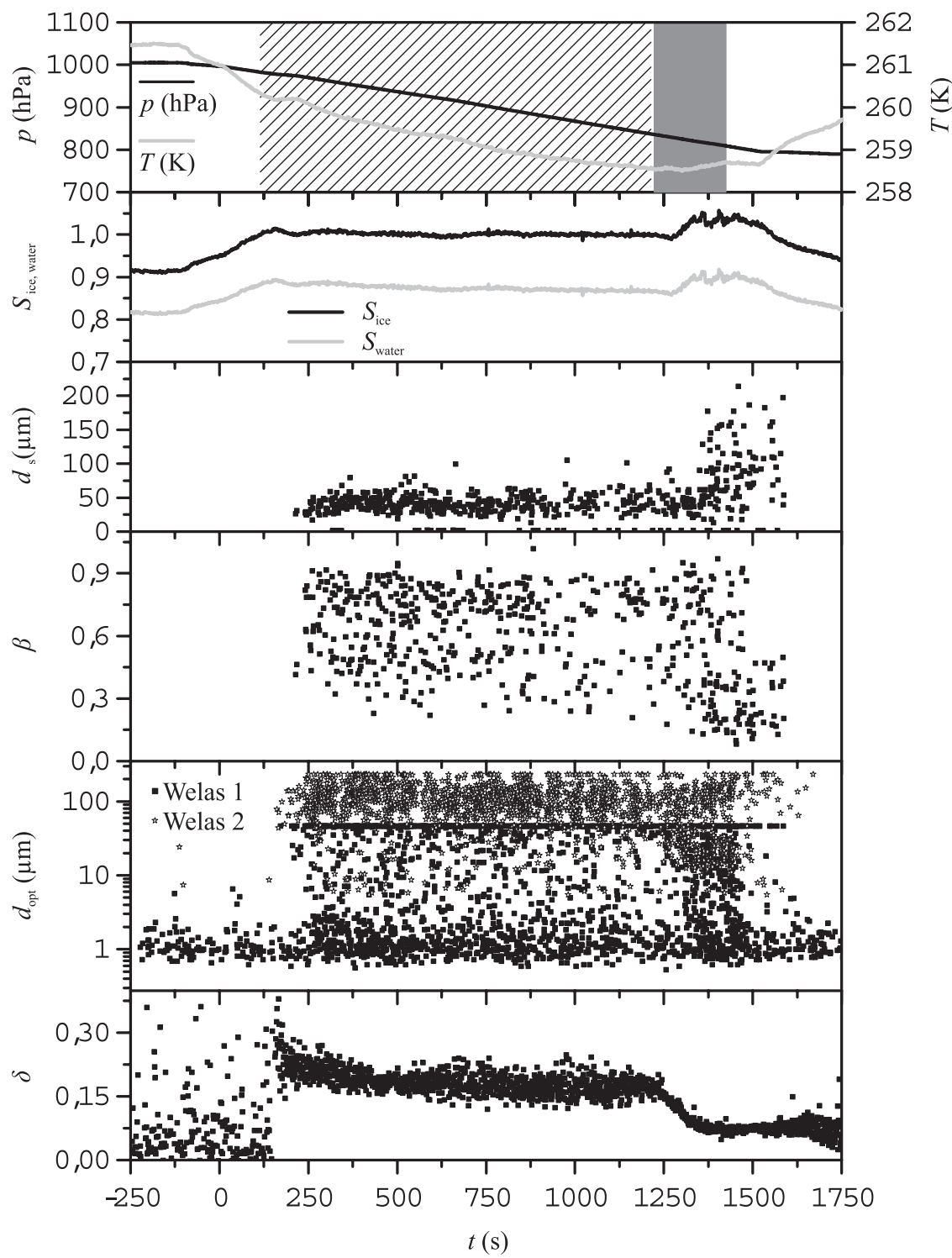

FIG. 16. The chronology of the AIDA experiment IN09_33 that was performed at temperatures between 258 and $262 \mathrm{~K}$. (from top to bottom) The pressure $p$, gas temperature $T$, saturation ratio with respect to ice $S_{\text {ice }}$ and water $S_{\text {water }}$, sphere-equivalent diameter $d_{s}$, roundness $\beta$, optical particle diameter measured by the two optical particle counters (Welas 1: $0.7-40 \mu \mathrm{m}$, Welas 2: $2.3-104 \mu \mathrm{m}$ ), and the depolarization $\delta$ are plotted as a function of the time $t$ elapsed during the experiment. (top) The periods when water droplets (gray) and ice seeds (hatched) had been injected are marked.

crystals are significantly overpredicted by the morphology diagram.

The PHIPS instrument presented in this paper served as a prototype of a more advanced version that is presently in the test phase. It is a novel combination between 1) a particle imager with the same excellent optical properties that have been demonstrated in this paper, but with the addition of dual-imaging optics viewing the same particle under two different angles to obtain 3D shape information and the particle orientation, and 2) a polar nephelometer that simultaneously measures the scattering phase function at $532 \mathrm{~nm}$ of the twofold-imaged ice crystals at 30 different angles: in the forward direction from $1^{\circ}$ to $10^{\circ}$ with an angular resolution of $1^{\circ}$, and with $8^{\circ}$ angular resolution between $18^{\circ}$ and $170^{\circ}$. A compact aircraft-ready version of the novel PHIPS instrument has also been designed and is currently under construction. It is planned to deploy the instrument in future missions of the new 


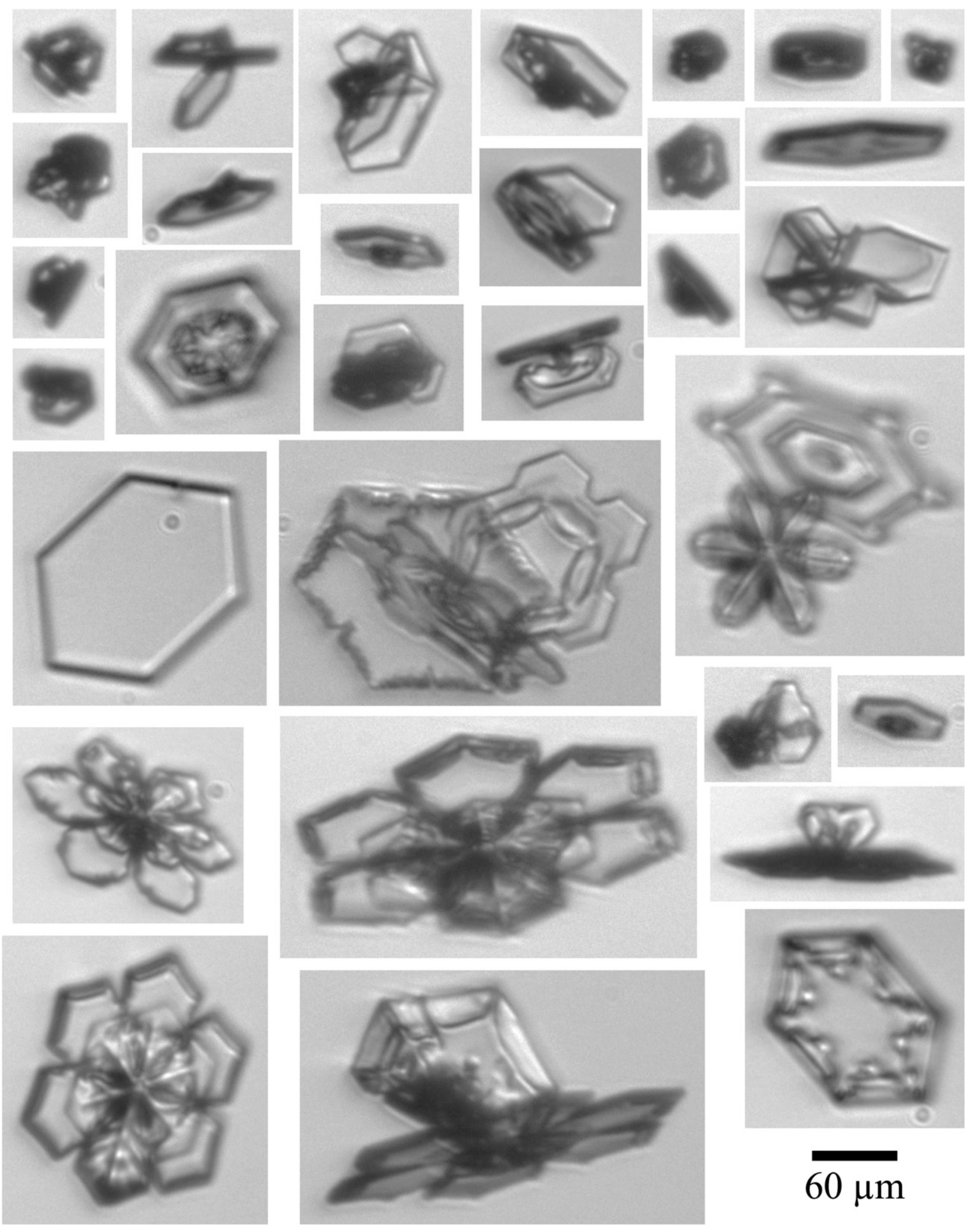

FIG. 17. A subjective selection of crystals that had been imaged during the experiment performed in the AIDA chamber at temperatures between 258 and $262 \mathrm{~K}$. The large and complex crystals in the lower part of the figure were observed after spraying water droplets into the chamber. 
German High Altitude and Long Range Research Aircraft (HALO).

A new image-processing algorithm is presently being developed, which corrects for the oversizing of out-offocus particles. It takes advantage of the full grayscale information of the PHIPS images. Furthermore, algorithms must be developed to combine the 3D information with the phase function measurements.

Acknowledgments. We express our gratitude to the technical staff of IMK-AAF. Without their ongoing support, this work would not have been possible. Further thanks go to P. Connolly for valuable hints and discussions. This work was funded within the Helmholtz Research Program Atmosphere and Climate and partly by the German Research Foundation (Contract SCHN 1140/1-1) within the framework of the Priority Program 1294.

\section{REFERENCES}

Amsler, P., O. Stetzer, M. Schnaiter, E. Hesse, S. Benz, O. Möhler, and U. Lohmann, 2009: Ice crystal habits from cloud chamber studies obtained by in-line holographic microscopy related to depolarization measurements. Appl. Opt., 48, 5811-5822.

Arnott, W. P., 1998: Analytical tools for cloudscope ice measurements. NASA Contract NAG 2-1104 Final Rep., 25 pp. [Available online at http://www.dri.edu/Projects/replica/cloudscope/ CS_Report.pdf.]

Bailey, M., and J. Hallet, 2004: Growth rates and habits of ice crystals between $-20^{\circ}$ and $-70^{\circ}$ C. J. Atmos. Sci., 61, $514-544$

- and —, 2009: A comprehensive habit diagram for atmospheric ice crystals: Confirmation from the laboratory, AIRS II, and other field studies. J. Atmos. Sci., 66, 2888-2899.

Benz, S., K. Megahed, O. Möhler, H. Saathoff, R. Wagner, and U. Schurath, 2005: T-dependent rate measurements of homogeneous ice nucleation in cloud droplets using a large atmospheric simulation chamber. J. Photochem. Photobiol. A, 176, 208-217.

Cannon, T. W., 1976: Imaging devices. Atmos. Technol., 8, 32-37.

Connolly, P. J., M. J. Flynn, Z. Ulanowski, T. W. Choularton, M. W. Gallagher, and K. N. Bower, 2007: Calibration of the cloud particle imager probes using calibration beads and ice crystal analogs: The depth of field. J. Atmos. Oceanic Technol., 24, 1860-1879.

Ebert, V., H. Teichert, C. Giesemann, H. Saathoff, and U. Schurath, 2005: Fasergekoppeltes In-situ-Laserspektrometer für den selektiven Nachweis von Wasserdampfspuren bis in den ppbBereich. Tech. Mess., 72, 23-30.

Field, P. R., O. Möhler, P. Connolly, M. Krämer, R. Cotton, A. J. Heymsfield, H. Saathoff, and M. Schnaiter, 2006: Some ice nucleation characteristics of Asian and Saharan desert dust. Atmos. Chem. Phys., 6, 2991-3006.

Freeman, H., 1974: Computer processing of line-drawing images. Comput. Surv., 6, 57-97.
Heymsfield, A. J., and L. M. Miloshevich, 2003: Parameterizations for the cross-sectional area and extinction of cirrus and stratiform ice cloud particles. J. Atmos. Sci., 60, 936-956.

Jähne, B., 2002: Digital Image Processing. 5th ed. Springer, 585 pp.

Kobayashi, T., 1961: The growth of snow crystals at low supersaturations. Philos. Mag., 6, 1363-1370.

Korolev, A., and G. Isaac, 2003: Roundness and aspect ratio of particles in ice clouds. J. Atmos. Sci., 60, 1795-1808.

Lawson, R. P., B. A. Baker, C. G. Schmitt, and T. L. Jensen, 2001: An overview of microphysical properties of Artic clouds observed in May and July 1998 during FIRE ACE. J. Geophys. Res., 106, 14 989-15 014.

Libbrecht, K. G., 2005: The physics of snow crystals. Rep. Prog. Phys., 68, 855-895.

McFarquhar, G. M., and A. J. Heymsfield, 1996: Microphysical characteristics of three anvils sampled during the Central Equatorial Pacific Experiment. J. Atmos. Sci., 53, 2401-2423.

Möhler, O., and Coauthors, 2003: Experimental investigation of homogeneous freezing of sulphuric acid particles in the aerosol chamber AIDA. Atmos. Chem. Phys., 3, 211-223.

- and Coauthors, 2006: Efficiency of the deposition mode ice nucleation on mineral dust particles. Atmos. Chem. Phys., 6, 3007-3021.

Ridler, T. W., and S. Calvard, 1978: Picture thresholding using an iterative selection method. IEEE Trans. Syst. Man Cybern., SCM-8, 630-632.

Sassen, K., 1991: The polarization lidar technique for cloud research: A review and current assessment. Bull. Amer. Meteor. Soc., 72, 1848-1866.

Sternberg, S. R., 1983: Biomedical image processing. IEEE Comp., 16, 22-34.

Ulanowski, Z., E. Hesse, P. H. Kaye, A. J. Baran, and R. Chandrasekhar, 2003: Scattering of light from atmospheric ice analogues. J. Quant. Spectrosc. Radiat. Transfer, 79-80, 1091-1102.

,,--- , and,- 2006 : Light scattering by complex iceanalogue crystals. J. Quant. Spectrosc. Radiat. Transfer, 100, 382-392.

—, P. H. Kaye, E. Hirst, and R. S. Greenaway, 2010: Light scattering by ice particles in the Earth's atmosphere and related laboratory measurements. Proc. 12th Int. Conf. Electromagnetic and Light Scattering XII, Helsinki, Finland, University of Helsinki and Cosponsors, 294-297. [Available online at http://www.helsinki.fi/els/articles/74/article.pdf.]

Wagner, R., C. Linke, K.-H. Naumann, M. Schnaiter, M. Vragel, M. Gangl, and H. Horvath, 2009: A review of optical measurements at the aerosol and cloud chamber AIDA. J. Quant. Spectrosc. Radiat. Transfer, 110, 930-949.

Weickmann, H., 1945: Formen und Bildung atmosphärischer Eiskristalle. Beitr. Phys. Atmos., 28, 12-52.

_ 1949: Die Eisphase in der Atmosphäre. Ber. Dtsch. Wetterdienstes U.S. Zone, 6, 3-54.

Wendisch, M., and Coauthors, 2005: Impact of cirrus crystal shape on solar spectral irradiance: A case study for subtropical cirrus. J. Geophys. Res., 110, D03202, doi:10.1029/2004JD005294

Zack, G. W., W. E. Rogers, and S. A. Latt, 1977: Automatic measurement of sister chromatid exchange frequency. J. Histochem. Cytochem., 25, 741-753.

Zhang, Y., A. Macke, and F. Albers, 1999: Effect of crystal size spectrum and crystal shape on stratiform cirrus radiative forcing. Atmos. Res., 52, 59-75. 